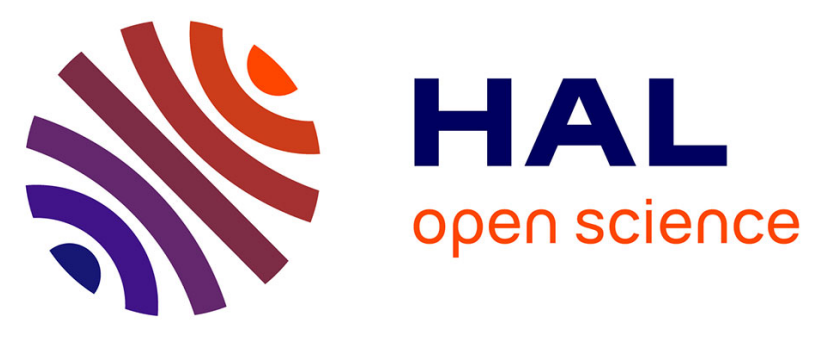

\title{
Interfacial Compatibilization in Ternary Polymer Nanocomposites: Comparing Theory and Experiments
}

Shawn M Maguire, Nadia M Krook, Patrice Rannou, Connor R Bilchak, Robert Brosnan, Andreea-Maria Pana, Manuel Maréchal, Kohji Ohno, Arthi Jayaraman, Russell J Composto, et al.

\section{To cite this version:}

Shawn M Maguire, Nadia M Krook, Patrice Rannou, Connor R Bilchak, Robert Brosnan, et al.. Interfacial Compatibilization in Ternary Polymer Nanocomposites: Comparing Theory and Experiments. Macromolecules, 2021, 54 (2), pp.797 - 811. 10.1021/acs.macromol.0c02345 . hal-03413336

\section{HAL Id: hal-03413336 https://hal.science/hal-03413336}

Submitted on 4 Nov 2021

HAL is a multi-disciplinary open access archive for the deposit and dissemination of scientific research documents, whether they are published or not. The documents may come from teaching and research institutions in France or abroad, or from public or private research centers.
L'archive ouverte pluridisciplinaire HAL, est destinée au dépôt et à la diffusion de documents scientifiques de niveau recherche, publiés ou non, émanant des établissements d'enseignement et de recherche français ou étrangers, des laboratoires publics ou privés. 


\title{
Interfacial Compatibilization in Ternary Polymer Nanocomposites: Comparing Theory and Experiments
}

\begin{abstract}
Shawn M. Maguire, ${ }^{\dagger, *}$ Nadia M. Krook,,${ }^{\ddagger *}$ Arjita Kulshreshtha, ${ }^{\S}$ Connor R. Bilchak, ${ }^{\dagger}$ Robert Brosnan, ${ }^{\dagger}$ Andreea-Maria Pana, ${ }^{\|}$Patrice Rannou, $\perp$ Manuel Maréchal,$\perp$ Kohji Ohno, ${ }^{\mathbb{T}}$ Arthi Jayaraman, ${ }^{*}{ }^{\S}$ Russell J. Composto $^{*, \dagger}$
\end{abstract}

†Department of Materials Science and Engineering, University of Pennsylvania, Philadelphia, PA, 19104

¥DuPont Co., Wilmington, DE, 19803

$\S^{\S}$ Department of Chemical and Biomolecular Engineering, University of Delaware, Newark DE, 19716

|| Department of Chemical and Biomolecular Engineering, University of Pennsylvania, Philadelphia, PA, 19104

$\perp$ Univ. Grenoble Alpes, CNRS, CEA, IRIG-SyMMES, 38000 Grenoble, France II Department of Polymer Chemistry, Kyoto University, Kyoto, Japan

Corresponding Authors

*E-mail: composto@ seas.upenn.edu

*E-mail: arthij@udel.edu 


\title{
For Table of Contents use only
}

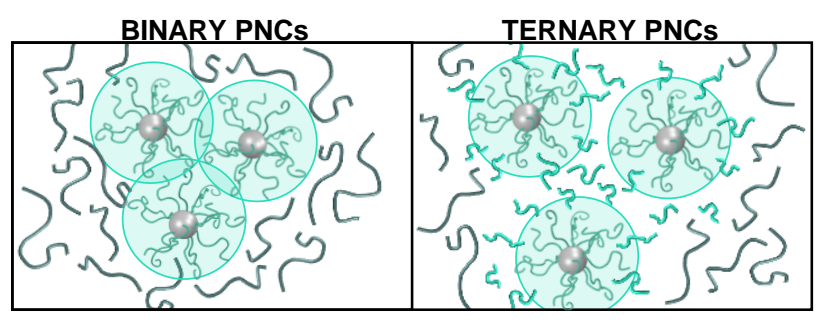

\begin{abstract}
In this work, we examine binary and ternary nanocomposites of poly(methyl methacrylate) grafted silica nanoparticles (PMMA-NP), in poly(styrene-ran-acrylonitrile) (SAN), and poly(methyl methacrylate) matrices as a platform to directly probe governing parameters guiding phase behavior and nanoparticle assembly in composite materials. Through the addition of PMMA matrix chains similar in molecular weight to the grafted PMMA chains and significantly smaller than the SAN matrix chains, we observe increased nanoparticle miscibility in off-critical compositions due to interfacial segregation of PMMA matrix chains. A simple interfacial model provides a general guideline for predicting the extent of compatibilization. Further insights on compatibilization behavior are provided by polymer particle pair correlation functions and structure factors obtained using Polymer Reference Interaction Site Model theory calculations as well as polymer concentration profiles from molecular dynamics simulations. This study serves as a guideline to facilitate PNC processing and design of materials for a broad range of technological applications.
\end{abstract}




\section{INTRODUCTION}

The ability to control the dispersion state of nano-sized inorganic or hybrid fillers in polymer nanocomposites (PNCs) has been a continuous challenge in the development of functional hybrid materials for a wide array of applications. Examples include the addition of rigid polymer nanoparticles (NP) to a liquid or polymer electrolyte for enhanced mechanical modulus without sacrificing ionic conductivity, ${ }^{1-3}$ the incorporation of gold nanorods to a polymer matrix for tuning optical absorption and polarization, ${ }^{4-6}$ or using polymer-grafted silica nanoparticles to create membranes with controllable separation capabilities. ${ }^{7}$ Since the bulk material properties of the composite are directly correlated with the spatial distribution of the nanoparticles within the polymer matrix, ${ }^{8-12}$ the ability to achieve uniform nanoparticle dispersion through different processing techniques, including self-assembly and external fields, has attracted considerable attention. While there have been significant advances made utilizing these methods, often these are non-equilibrium processes, resulting in composites having metastable morphologies. These kinetically trapped PNCs are therefore unsuitable for applications that operate at high temperatures, which accelerate NP motion and instability, leading to phase separation and degradation. Therefore, finding ways to increase the miscibility windows of these composites at elevated temperatures is enticing for industrial applications that rely on controlled spatial distributions of NPs within the polymer phase.

Previously, it has been shown by Schmitt et al. that a binary PNC system of silica particles grafted with polystyrene (PS) and PMMA ligands with an upper critical solution temperature (UCST) can impart phase behavior comparable to analogous linear polymer blends. ${ }^{13}$ However, while the trends in phase behavior between the two systems were similar, the PNC demonstrated increased miscibility and reduced kinetics of domain growth. The apparent 
increase in miscibility was rationalized as a consequence of a reduced thermodynamic driving force for phase separation by decreasing the number of unfavorable segmental interactions between each ligand. Additionally, the presence of NP cores resulted in a smaller number density of segmental contacts. ${ }^{13}$ Similarly, work by Karim and co-workers compared the phase separation behavior of a system of gold nanoparticles grafted with PS (AuPS) inside a PMMA matrix to that of a binary homopolymer blend composed of PS and PMMA. ${ }^{14}$ Comparison of the surface topologies of the two systems revealed a lower interfacial tension between AuPS and PMMA than that in the homopolymer blend. This difference was attributed to the sterically hindered interactions of PMMA matrix chains with the chains grafted to the surface of the nanoparticle. Taken together, these studies offer insights into potential routes of facilitating PNC miscibility, specifically, by focusing on the interface between the grafted nanoparticle and matrix.

To probe if the PNC miscibility can be expanded via increased interfacial compatibilization, a polymer system has to be judiciously chosen such that the phase behavior of the homopolymer blend is documented and has an accessible critical point for the tracking of changes in miscibility. The binary homopolymer blend of poly(styrene-ran-acrylonitrile) (SAN, 33 wt.\% AN) and PMMA has been shown to have an accessible lower critical solution temperature (LCST) of $160^{\circ} \mathrm{C}$ that can be tuned through changing the composition of SAN..$^{15-19}$ The addition of nanoparticles to the PMMA/SAN blend has been demonstrated as a way to further manipulate both the phase behavior and phase separation temperature of the system. ${ }^{20-25}$ While the aforementioned studies focused on UCST PNCs with unfavorable segmental interactions below a critical temperature, the favorable interactions below a critical temperature of the PMMA/SAN blend with LCST phase behavior allows for direct validation of the proposed 
interfacial tension and limited segmental interaction theories. Previously, favorable interactions between SAN graft polymers and PMMA matrix polymers in PNCs containing SAN grafted silica nanoparticles have been shown to promote uniform dispersion where chosen design parameters like large particle size, small molecular weight of graft chains and high molecular weight of matrix chains favored phase separation. ${ }^{26}$ However, PNCs with graft-matrix interactions that follow LCST phase behavior, exhibiting favorable segmental interactions at low temperatures that become progressively weaker and eventually repulsive as temperature increases, show a sharp dispersion to aggregation transition on increasing temperature as seen for polystyrene (PS) grafted silica particles in poly (vinyl methyl ether) (PVME) matrix in past experiments and simulations. ${ }^{27}$ Accordingly, through the addition of PMMA homopolymer to a binary PMMA-NP/SAN (i.e. LCST composite), the number of favorable segmental interactions between each chemical species can be restored to reduce the thermodynamic driving force for phase separation at high temperatures.

In the present work, we examine binary and ternary nanocomposites of poly(methyl methacrylate) grafted silica nanoparticles (PMMA-NP) mixed with SAN (binary), and PMMANPs, SAN, and PMMA homopolymer (ternary), as model systems to directly probe the governing parameters guiding phase behavior and nanoparticle assembly. As-cast PNC films are characterized as a function of nanoparticle loading, revealing well dispersed NP systems. Phase behavior of the binary and ternary PNC was investigated between 155 and $195^{\circ} \mathrm{C}$ and characterized using a combination of cloud point observations, transmission electron microscopy (TEM), and small-angle X-ray scattering (SAXS). For both binary and ternary PNCs, crosssectional TEM micrographs reveal a 3-stage transition from discrete to string-like to bicontinuous NP domains with increasing NP loadings when annealed in the two-phase regime. 
The scattering profiles obtained through SAXS reveal the emergence of two distinct length scales underpinning the binary and ternary PNC phase behavior — specifically, a smaller lengthscale feature representing the average center-to-center interparticle distance as well as a larger length-scale feature representing the scattering between nanoparticle clusters. Modeling of these two scattering features allows for the accurate capture of the phase transition within the binary and ternary PNCs. By compiling the cloud point, TEM, and SAXS results, we demonstrate that the addition of 5 wt.\% PMMA homopolymer to the binary PNC stabilizes the composite material through directed segregation of PMMA to the PMMA-NP/SAN interface, leading to increased miscibility in off-critical compositions. To explain this compositional dependence, a simple interfacial scaling model is derived, providing a general guideline for predicting the extent of compatibilization. Polymer Reference Interaction Site Model (PRISM) theory calculations and molecular dynamics (MD) simulations based on a coarse-grained (CG) model are performed to elucidate the effect of the amount of added PMMA matrix (quantified in terms of volume fraction) on PNC structure. Experimental plots of the composite structure factors, which show a dispersion to aggregation transition in PNCs on increasing temperature, are compared to particleparticle structure factors obtained using PRISM theory to verify the ability of the CG model to capture experimental phase behavior. Thereafter, MD simulations based on the CG model are used to calculate concentration profiles for graft and matrix polymers in the vicinity of the particle at various PMMA matrix volume fractions. Results from MD simulations show that when PMMA graft and SAN matrix interactions are unfavorable (at high temperatures), PMMA matrix concentration increases near the particle surface, suggestive of compatibilization. Further, particle-particle pair correlation functions and particle structure factor plots from PRISM theory 
show that increased volume fraction of PMMA matrix chains leads to a reduced tendency for aggregation in PNCs at high temperatures.

\section{EXPERIMENTS}

Materials. Poly(styrene-ran-acrylonitrile) $\left(\mathrm{SAN}, \mathrm{M}_{\mathrm{w}}=118 \mathrm{~kg} / \mathrm{mol}, \mathrm{M}_{\mathrm{w}} / \mathrm{M}_{\mathrm{n}}=2.24, \mathrm{~T}_{\mathrm{g}}=114{ }^{\circ} \mathrm{C}\right.$, containing 33 wt.\% acrylonitrile) was provided by Monsanto and purified twice by adding a solution of SAN and chloroform ( $\geq 99.9 \%$, for HPLC) into methanol ( $\geq 99.9 \%$, for HPLC) at a 1:10 volume ratio. After allowing SAN to precipitate for $1 \mathrm{~h}$, the solvent was removed, and the precipitant was dried for $48 \mathrm{~h}$. Once dried, the SAN was redissolved in chloroform and precipitated with methanol once more. Poly(methyl methacrylate) (PMMA, $\mathrm{M}_{\mathrm{w}}=21 \mathrm{~kg} / \mathrm{mol}$, $\mathrm{M}_{\mathrm{w}} / \mathrm{M}_{\mathrm{n}}=1.14, \mathrm{~T}_{\mathrm{g}}=110^{\circ} \mathrm{C}$ ) was purchased from Polymer Source Inc. and used as received. Silica nanoparticles (15 nm diameter) grafted with PMMA brushes, denoted as PMMA-NPs, were prepared using surface-initiated atom transfer radical polymerization. ${ }^{28}$ The brush weightaverage molecular weight and grafting density were $19 \mathrm{~kg} / \mathrm{mol}$ and 0.7 chains $/ \mathrm{nm}^{2}$, respectively, as determined by thermogravimetric analysis (TGA, TA Instruments Q600 SDT) at a heating rate of $10^{\circ} \mathrm{C} / \mathrm{min}$ under argon flow between the temperatures of $25^{\circ} \mathrm{C}$ and $550^{\circ} \mathrm{C}$. The glass transition temperature $\left(\mathrm{T}_{\mathrm{g}}\right)$ of each polymer was measured using a differential scanning calorimeter (DSC, TA Instruments Q2000) between the temperatures of $25^{\circ} \mathrm{C}$ and $150^{\circ} \mathrm{C}$. In all cases, two heating/cooling cycles were performed at a heating/cooling rate of $5^{\circ} \mathrm{C} / \mathrm{min}$. The $\mathrm{T}_{\mathrm{g}}$ values were obtained from the second heating in order to eliminate any thermal history. Details of the TGA and DSC characterization of PMMA, SAN and PMMA-NPs are given in the Supporting Information. EpoxiCure 2 epoxy hardener and epoxy resin were purchased from Buehler. Ntype, $<100>$ oriented silicon wafers (dopant $\mathrm{Ph}, 10-20 \Omega \cdot \mathrm{cm}$ resistivity, 475-575 $\mu \mathrm{m}$ thickness, single side polished) were purchased from Silicon Quest International. P-type, $<100>$ oriented 
silicon wafers (dopant $\mathrm{B}, 0.001-0.005 \Omega \cdot \mathrm{cm}$ resistivity, $500 \mu \mathrm{m}$ thickness, single side polished) with a $300 \mathrm{~nm}$ wet thermal oxide layer were purchased from University Wafer.

Cloud Point Observations. Phase diagrams for the binary and ternary PNCs were first mapped qualitatively via cloud point observations. This was defined as the temperature at which the film changed from transparent to opaque when viewed against a black background. Polymer solutions with varying compositions were dissolved in methyl isobutyl ketone (MIBK, $\geq 98.5 \%$, Certified ACS, Fisher Chemical) and stirred for $24 \mathrm{~h}$ using a magnetic stir bar. The solutions (17 wt. $\%$ in MIBK) were spin-coated (1000 rpm, 60 seconds) onto $1 \mathrm{~cm}^{2}$ glass substrates (washed with methanol and toluene and dried under nitrogen flow) and dried at $105^{\circ} \mathrm{C}$ for $1 \mathrm{~h}$ to remove residual solvent. All films were smooth and homogeneous as-cast. Film thickness, measured with reflectometry (Filmetrics F3-UV), ranged from 2000-2500 nm depending on the composite composition. Samples were subsequently annealed to the desired temperature on a hot stage (Mettler FP-82, Mettler Toledo, INC.) under continuous argon flow and immediately quenched to room temperature after 24 and $72 \mathrm{~h}$ of annealing.

Small-Angle X-ray Scattering (SAXS). To prepare samples for small-angle X-ray scattering, PNC solutions (17 wt.\% in MIBK) were spin-coated (1000 rpm, 60 seconds) onto $1 \mathrm{~cm}^{2}$ silicon wafers with a $300 \mathrm{~nm}$ thermal oxide layer and then dried at $105^{\circ} \mathrm{C}$ for $1 \mathrm{~h}$ to remove residual solvent. Following drying, the samples were annealed on a hot stage for varying amounts of time under continuous argon flow and then immediately quenched to room temperature. To lift the films, the edges of the substrate were scored and then floated on a 1:5 vol\% solution of $\mathrm{NaOH}$ $(50 \% \mathrm{w} / \mathrm{w} \mathrm{NaOH})$ and deionized water $\left(\mathrm{DI} \mathrm{H}_{2} \mathrm{O}\right)$. After lifting from its substrate, the films were transferred to pure DI $\mathrm{H}_{2} \mathrm{O}$ and then onto $2 \times 2 \mathrm{~cm}^{2}$ Kapton ${ }^{\circledR}$ films. In order to achieve a sufficient signal-to-noise ratio, 4 films were stacked on top of one another, resulting in a film 
stack thickness of $c a .10 \mu \mathrm{m}$. The films were allowed to dry for 6 hours at room temperature between each sequential stack. SAXS measurements were performed on a Xenocs Xeuss 2.0 at the University of Pennsylvania, with a sample-to-detector distance of $6.36 \mathrm{~m}$ with a $\mathrm{Cu}-\mathrm{K}_{\alpha}$ source, providing an available q-range of 0.003-0.09 $\AA^{-1}$. Raw two-dimensional scattering images were collected on a Pilatus $1 \mathrm{M}$ detector for 20 minutes per sample and were then azimuthally integrated into one dimensional patterns for analysis using Foxtrot software.

Transmission Electron Microscopy (TEM). To prepare the samples for electron microscopy characterization, the floated films (see SAXS Methods) were lifted from the liquid-air interface of the $\mathrm{NaOH}$ solution, transferred onto Teflon ${ }^{\circledR}$, and sputter-coated with a gold and palladium layer (a guide for the eye during microscopy). After sputter-coating, the specimens were lifted from the Teflon ${ }^{\circledR}$ and embedded in two-part epoxy. Once the epoxy cured, ca. $70 \mathrm{~nm}$ crosssections of the PNCs were prepared by ultramicrotomy (Leica Ultracut S Ultramicrotome) with a room temperature diamond knife. TEM characterization of the ultramicrotomed cross-sections, transferred onto carbon coated TEM grids, was performed with a JEOL JEM-1400 TEM at 120 $\mathrm{kV}$. Radial autocorrelation functions of TEM images were produced to discern the average center-to-center interparticle distances (ID) of the grafted nanoparticles in as-cast films using Gwyddion software.

\section{COMPUTATIONS}

Coarse-grained (CG) model. Graft and matrix polymers (denoted with subscripts G and M, respectively) were modeled as bead-spring ${ }^{29}$ chains with harmonic bonds in CG simulations. The PMMA graft (G) and PMMA matrix chains (matrix_2, M2) were comprised of $\mathrm{N}_{\mathrm{G}}$ and $\mathrm{N}_{\mathrm{M} 2}$ number of CG beads, where each bead of diameter $1 d$ was equal to the Kuhn segment length of PMMA $(1.53 \mathrm{~nm})$. The first bead of each graft chain is tethered to the nanoparticle of size $\mathrm{D}_{\mathrm{p}}$ via 
a surface grafting site. The number of grafting sites on the nanoparticle was chosen based on the particle size and the desired surface grafting density. The SAN matrix chains (matrix_1, M1) were comprised of $\mathrm{N}_{M}$ number of CG beads, each bead of diameter $1 d$ as the Kuhn segment of SAN is similar to that of PMMA. In all chains the harmonic bonds were modeled using the form

$$
\mathrm{U}_{\mathrm{bond}}(\mathrm{r})=\mathrm{k}_{\mathrm{bond}}\left(\mathrm{r}-\mathrm{r}_{\mathrm{o}}\right)^{2}
$$

with the force constant $\mathrm{k}_{\text {bond }}=50 \mathrm{kT} / d^{2}$ and the equilibrium bond distance $\mathrm{r}_{0}=1 d$. No angle restrictions were imposed on the graft and matrix CG beads to model flexible graft and matrix chains.

Pairwise non-bonded interactions between all graft and matrix bead pairs (GG, GM1, and M1M1 for binary PNCs and GG, GM1, GM2, M1M1, M1M2, and M2M2 for ternary PNCs) were modeled using the cut and shifted Lennard Jones ${ }^{30}(\mathrm{LJ})$ interaction potential given by

$$
U_{i j}(r)=\left\{\begin{array}{r}
4 \varepsilon_{i j}\left[\left(\frac{\sigma_{i j}}{r}\right)^{12}-\left(\frac{\sigma_{i j}}{r}\right)^{6}\right]+\varepsilon_{i j} ; r \leq 2.0 \sigma_{i j} \\
0 \quad ; r>2.0 \sigma_{i j}
\end{array}\right.
$$

with $\sigma_{\mathrm{ij}}=1 d$ for all pairs. In the case of binary PNCs, $\varepsilon_{\mathrm{ii}}=0.1 \mathrm{kT}$ for all like beads pairs (GG, $\mathrm{M} 1 \mathrm{M} 1)$ and $\varepsilon_{\mathrm{GM} 1}$ is varied from $0.2-0.07 \mathrm{kT}$, thereby tuning the graft-matrix interaction from being energetically favorable $\left(\varepsilon_{\mathrm{GM} 1}=0.2 \mathrm{kT}\right)$, to athermal $\left(\varepsilon_{\mathrm{GM} 1}=0.1 \mathrm{kT}\right)$, and finally unfavorable at $\varepsilon_{\mathrm{GM} 1}=0.09 \mathrm{kT}$ and $0.07 \mathrm{kT}$. Thus, the LCST transition for graft (PMMA) and matrix_1 (SAN) polymer chemistries with increasing temperature is captured by decreasing $\varepsilon_{\mathrm{GM} 1}$ in the model. Accordingly, PNCs with $\varepsilon_{\mathrm{GM} 1}=0.2 \mathrm{kT}$ correspond to PNCs at low temperatures in experiments (below LCST), while PNCs with $\varepsilon_{\mathrm{GM} 1}=0.1 \mathrm{kT}$ and $\varepsilon_{\mathrm{GM} 1}=0.07 \mathrm{kT}$ correspond to PNCs at intermediate and high temperatures in experiments, respectively. In the case of ternary PNCs, the GG and M1M1 bead pairwise interactions were similar to the binary PNCs. With G 
and M2 beads representing the same chemistry, the $\varepsilon_{\mathrm{ij}}$ for all $\mathrm{G}$ bead pairwise interactions were identical to $\mathrm{M} 2$ bead pairwise interactions (i.e., $\varepsilon_{\mathrm{GG}}=\varepsilon_{\mathrm{M} 2 \mathrm{M} 2}=0.1 \mathrm{kT}, \varepsilon_{\mathrm{GG}}=\varepsilon_{\mathrm{GM} 2}=0.1 \mathrm{kT}, \varepsilon_{\mathrm{GM} 1}=$ $\varepsilon_{\mathrm{M} 2 \mathrm{M} 1}$ ). Lastly, all pairwise interactions involving the nanoparticle (with pairs ij $=\mathrm{PP}, \mathrm{PG}, \mathrm{PM} 1$, and PM2) were modeled as purely repulsive using the Weeks Chandler Andersen (WCA) ${ }^{31}$ potential with $\varepsilon_{\mathrm{ij}}=0.5 \mathrm{kT}$ and $\sigma_{\mathrm{ij}}$ equal to the mean diameter of $\mathrm{ij}$ beads pairs.

Parameters Varied. The relative lengths of graft and matrix polymer chains were based on relative molecular weights for PMMA and SAN polymers used in experiments (Table S1). We selected $\mathrm{N}_{\mathrm{G}}=15$ and $\mathrm{N}_{\mathrm{M} 1}=75$ for both binary and ternary cases. In the case of ternary PNCs, the matrix polymer was composed of two types of polymer matrix chains: $\mathrm{N}_{\mathrm{M} 1}=75$ and $\mathrm{N}_{\mathrm{M} 2}=15$. The size of the nanoparticle, $\mathrm{D}_{\mathrm{P}}=5 d$, and surface grafting density of graft polymer chains, $\Sigma=$ 0.76 chains $/ d^{2}$, were maintained constant for all PNCs. We note that in the computations, the above choices of particle diameter and graft and matrix chain lengths were smaller than those in experiments, but maintained a similar ratio of matrix to graft chain length, particle curvature, and grafted monomer crowding as in the case of experiments (see Table S1). For all cases, the fraction of any volume occupied by particle, graft and matrix (M1 and/or M2) beads is kept at $\eta$ $=0.36$; this packing fraction approaches the behavior of an incompressible melt-like polymer matrix. PNC composition was defined in terms of two parameters; the grafted NP filler fraction,

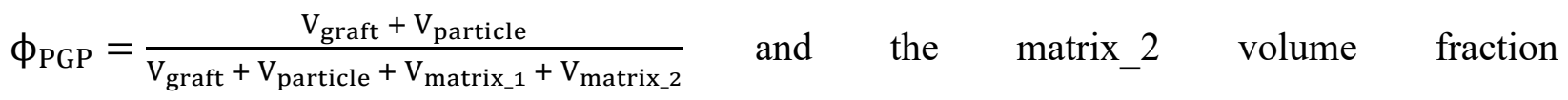
$\phi_{\mathrm{M} 2}=\frac{V_{\text {matrix_2 }}}{V_{\text {graft }}+V_{\text {particle }}+V_{\text {matrix_1 }}+V_{\text {matrix_2 }}}$. For binary and ternary PNCs, the SAN polymer matrix volume fraction is $1-\phi_{\mathrm{PGP}}-\phi_{\mathrm{M} 2}=\phi_{\mathrm{M} 1}$. 
Molecular Dynamics (MD) Simulations. The LAMMPS ${ }^{32}$ package was leveraged to perform the MD simulations with the CG model for PNCs (vide supra). For all simulations, the starting configuration consisted of a nanoparticle of size $\mathrm{D}_{\mathrm{P}}=5 d$ grafted with 60 chains, leading to a grafting density of 0.76 chains $/ d^{2}$. The grafted chains were relaxed from the starting configuration during a short simulation run with all (GG and PG) interactions set as purely repulsive using the WCA potential. The relaxed grafted nanoparticle configuration was placed in a large box $(\eta \sim 0.006)$, with $\phi_{\mathrm{PGP}}=0.01$, along with matrix chains with the desired composition defined by the $\phi_{\mathrm{M} 2}$ value. The large simulation box volume allowed mixing of graft and matrix chains in the NVT ensemble for $5 \times 10^{6}$ time steps, at a reduced temperature of $\mathrm{T}^{*}=1$ maintained using the Nose-Hoover ${ }^{33,34}$ thermostat with a damping time of $1 \tau$. In this mixing stage, the equations of motion were integrated using the velocity Verlet algorithm with time step $\Delta t=0.001 \tau$. The cubic simulation box was then shrunk isotropically in all three dimensions over $2 \times 10^{6}$ time steps to achieve a final simulation box volume packing fraction of $\eta=0.36$. At the final box size, PNC configurations were further equilibrated for $1 \times 10^{7}$ time steps with $\Delta t=$ $0.005 \tau$, and equilibrated configurations were then used to generate production run configurations every $10^{5}$ time steps for an additional $2 \times 10^{7}$ time steps to perform structural analyses.

MD simulations of a single grafted nanoparticle in a polymer matrix with a low $\phi_{\mathrm{PGP}}=$ 0.01 were performed with $\phi_{\mathrm{M} 2}$ ranging from 0 (binary case with matrix chain length $\mathrm{N}_{\mathrm{M} 1}=75$ ) to $\phi_{\mathrm{M} 2}=0.043$ and 0.1 (ternary cases with a low volume fraction of matrix_2 chains similar to matrix_2 composition used in experiments, see Table S2). An additional case of $\phi_{\mathrm{M} 2}=0.99$ $(\approx 1)$ corresponding to a binary PNC case with the matrix entirely composed of matrix_2 chains of length $\mathrm{N}_{\mathrm{M} 2}=15$ (and $\phi_{\mathrm{M} 1}=0$ ) was run as well. Additionally, for PNC cases of experimental 
relevance with higher grafted filler fractions of $\phi_{\mathrm{PGP}}=0.039,0.079$ and 0.205 and with aforementioned $\phi_{\mathrm{M} 2}$ values, Polymer Reference Interaction Site Model (PRISM) ${ }^{35}$ theory calculations described below were performed. This combination of MD simulations and PRISM theory enables calculation of structure factors at large length scales (i.e., at small scattering wave vector, q) with a significantly lower computational expense than MD simulations by eliminating the need to run large system sizes of multiparticle PNCs with high $\phi_{\text {PGP. }}$ Furthermore, unlike MD simulations, PRISM theory results are devoid of artifacts arising from chosen simulation box/system sizes and/or kinetic trapping effects.

Polymer Reference Interaction Site Model (PRISM) theory. Polymer Reference Interaction Site Model (PRISM) $)^{35-37}$ theory is a liquid-state theory that describes structure in polymer solutions and melts using the Ornstein-Zernike ${ }^{38}$ like mathematical form:

$$
\widehat{H}(q)=\widehat{\Omega}(q) \widehat{C}(q)[\widehat{\Omega}(q)+\widehat{H}(q)]
$$

Here, $\widehat{H}(q)$ is an $N x N$ matrix comprised of the total intermolecular pair correlations, $\widehat{C}(q)$ is an $\mathrm{N} \times \mathrm{N}$ matrix comprised of direct pair correlations, and $\widehat{\Omega}(\mathrm{q})$ is an $\mathrm{N} \times \mathrm{N}$ matrix comprised of intramolecular pair correlations. $\mathrm{N}$ is the number of interaction site types, which in this study can be 3 or 4 types of interaction sites corresponding to particle, graft, matrix_1 and/or matrix_2 beads depending on the chosen matrix composition $\left(\phi_{\mathrm{M} 2}=0,0.043,0.1\right.$ and 1$)$ for PNCs. Although graft and matrix_2 beads refer to the same chemistry (PMMA), they are designated as separate types of interaction sites since the intramolecular correlations for tethered graft chains are expected to be significantly different from matrix_2 chains.

The intramolecular pair correlation matrix, $\widehat{\Omega}(\mathrm{q})$, given as an input to PRISM theory, is composed of intramolecular structure factor elements, $\widehat{\omega_{X Y}}(q)$, for site pairs $X Y$ in each molecule. At any PNC grafted filler fraction $\left(\phi_{\mathrm{PGP}}=0.039, \phi_{\mathrm{PGP}}=0.079\right.$ and $\left.\phi_{\mathrm{PGP}}=0.205\right)$ 
and matrix_2 filler fraction $\left(\phi_{\mathrm{M} 2}=0,0.043,0.1,1\right), \widehat{\omega_{\mathrm{XY}}}(\mathrm{q})$ was approximated ${ }^{35}$ from single particle PNC MD simulations at low $\phi_{\mathrm{PGP}}=0.01$ and low $\phi_{\mathrm{M} 2}=0.043$ as follows:

$$
\widehat{\omega_{X Y}}(q)=\left\langle\frac{1}{N_{X}+N_{Y}} \sum_{i=1}^{N_{Y}} \sum_{j=1}^{N_{X}} \frac{\sin \left(q r_{i j}\right)}{q r_{i j}}\right\rangle
$$

where $\mathrm{N}_{X}$ and $\mathrm{N}_{Y}$ are the total number of interaction sites of type $\mathrm{X}$ and type $\mathrm{Y}$, respectively, within the same molecule, and $r_{i j}$ is the inter-site distance.

The PRISM equation is numerically solved for total intermolecular pair correlation, $\widehat{\mathrm{H}}(\mathrm{q})$ and direct pair correlation matrix, $\widehat{\mathrm{C}}(\mathrm{q})$. Each element of the $\widehat{\mathrm{C}}(\mathrm{q})$ matrix is a pairwise direct site correlation function, $\mathrm{c}_{\mathrm{XY}}$, expressed using a closure equation, in terms of the interaction potential, $\mathrm{U}_{\mathrm{XY}}$, and the total intermolecular pair correlation, $\mathrm{h}_{\mathrm{XY}}$, of any site pair $\mathrm{XY}$. For particle-particle pair, hypernetted chain closure $(\mathrm{HNC})^{39,40}$ was used as follows:

$$
c_{P P}(r)=h_{P P}(r)-\ln \left[h_{P P}(r)+1\right]-\beta U_{P P}(r), r>D_{P}
$$

For the remaining site pairs, XY (GG, GM1, GM2, M1M1, M1M2, M2M2, PG, PM1, and PM2), Percus Yevick (PY) $)^{35,37,41,42}$ closure was used as

$$
\begin{aligned}
& c_{X Y}(r)=\left(1-e^{\beta U_{X Y}(r)}\right)\left(h_{X Y}(r)+1\right), \quad r>d_{X Y} \\
& \left(h_{X Y}(r)+1\right)=g_{X Y}(r)=0, \quad r \leq d_{X Y}
\end{aligned}
$$

with $d_{X Y}$ equal to the mean diameter of sites $\mathrm{X}$ and $\mathrm{Y}$.

For a given input, the output in the form of pairwise total intermolecular correlations for all pairs of sites, in real and Fourier space was obtained upon numerically solving equations (3), (5) and (6) using the pyPRISM ${ }^{43}$ package.

Analyses. The spatial arrangement of graft, matrix_1 and matrix_2 beads as a function of distance from the particle surface was quantified from configurations sampled in MD simulation trajectories in the form of concentration profiles defined as 


$$
\mathrm{C}_{\mathrm{i} \mid \mathrm{r}}=<\frac{\mathrm{n}_{\mathrm{i}}(\mathrm{r})}{4 \pi \mathrm{r}^{2} \Delta \mathrm{r}}>
$$

where i refers to the bead type $(G, M 1$, or $M 2)$ and $n_{i}$ refers to the number of beads of type $i$ within a shell volume of thickness $\Delta \mathrm{r}$ at a distance $\mathrm{r}$ from the grafted nanoparticle surface. To plot the graft and matrix concentration profiles, $C_{i \mid r}$ was normalized with $C_{i \mid r}$ at $r=0$ (surface) for graft beads and $\mathrm{C}_{\mathrm{i} \mid \mathrm{r}}$ at $\mathrm{r}=\infty$ (bulk) for matrix_1 and matrix_2 beads. This normalized fraction is denoted as $\mathrm{C}_{\mathrm{i} \mid \mathrm{r}} / \mathrm{C}_{\mathrm{i} \mid \mathrm{r}=0}$ and $\mathrm{C}_{\mathrm{i} \mid \mathrm{r}} / \mathrm{C}_{\mathrm{i} \mid \mathrm{r}=\infty}$ for graft and matrix beads, respectively.

The thickness of the graft polymer layer on the nanoparticle surface was quantified by calculating the ensemble averaged grafted brush height, $\mathrm{H}_{\mathrm{b}}$, from the MD simulation configurations, defined as

$$
\mathrm{H}_{\mathrm{b}}=<\sqrt{\frac{\sum_{1}^{\mathrm{n}_{\mathrm{G}} \mathrm{r}_{\mathrm{i}}^{2}}}{\mathrm{n}_{\mathrm{G}}}}>
$$

with $r_{i}$ equal to the distance of an $\mathrm{i}$-th graft polymer bead from the surface of the particle and $n_{G}$ equal to the total number of graft polymer beads on the particle (i.e., $\mathrm{N}_{\mathrm{G}}$ times the number of graft chains on the particle). All quantities from MD simulations are reported as block averages obtained from 4 blocks of 50 configurations each and the error bars are standard deviations in the average values of the 4 blocks.

The structural output from PRISM theory calculations include the total intermolecular correlation matrix, $\widehat{H}(q)$, with the elements of the matrix $h_{X Y}(q)$ for a site pair $X Y$ converted to real space counterparts, $h_{X Y}(r)$. The intermolecular pair correlation function in real space, $\mathrm{g}_{\mathrm{XY}}(\mathrm{r})$, is calculated from $\mathrm{h}_{\mathrm{XY}}(\mathrm{r})$ as follows

$$
g_{X Y}(r)=h_{X Y}(r)+1
$$

In Fourier space, the structure factor matrix, $\widehat{S}(q)$, is obtained using $\widehat{H}(q)$ and $\widehat{\Omega}(q)$ as 


$$
\widehat{S}(q)=\widehat{H}(q)+\widehat{\Omega}(q)
$$

The elements of the structure factor matrix, include the particle-particle structure factor, $\operatorname{SPP}(\mathrm{q})$, and graft-graft structure factor, $\mathrm{S}_{\mathrm{GG}}(\mathrm{q})$. 


\section{RESULTS AND DISCUSSION}

\section{i.) As-cast Binary and Ternary PNCs}

a.
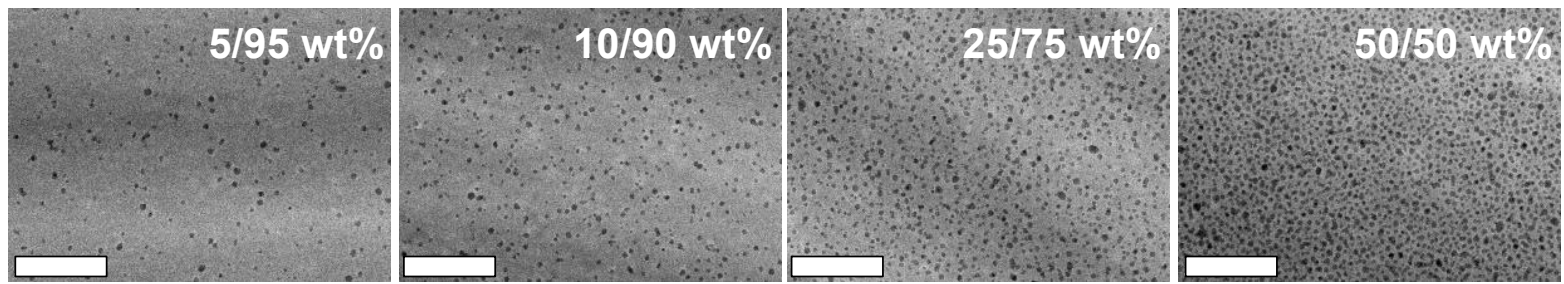

b.
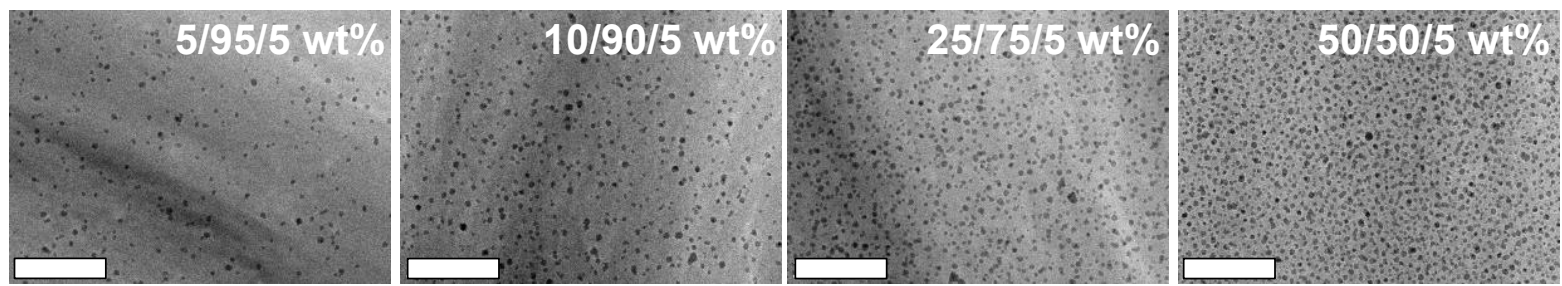

C.

d.
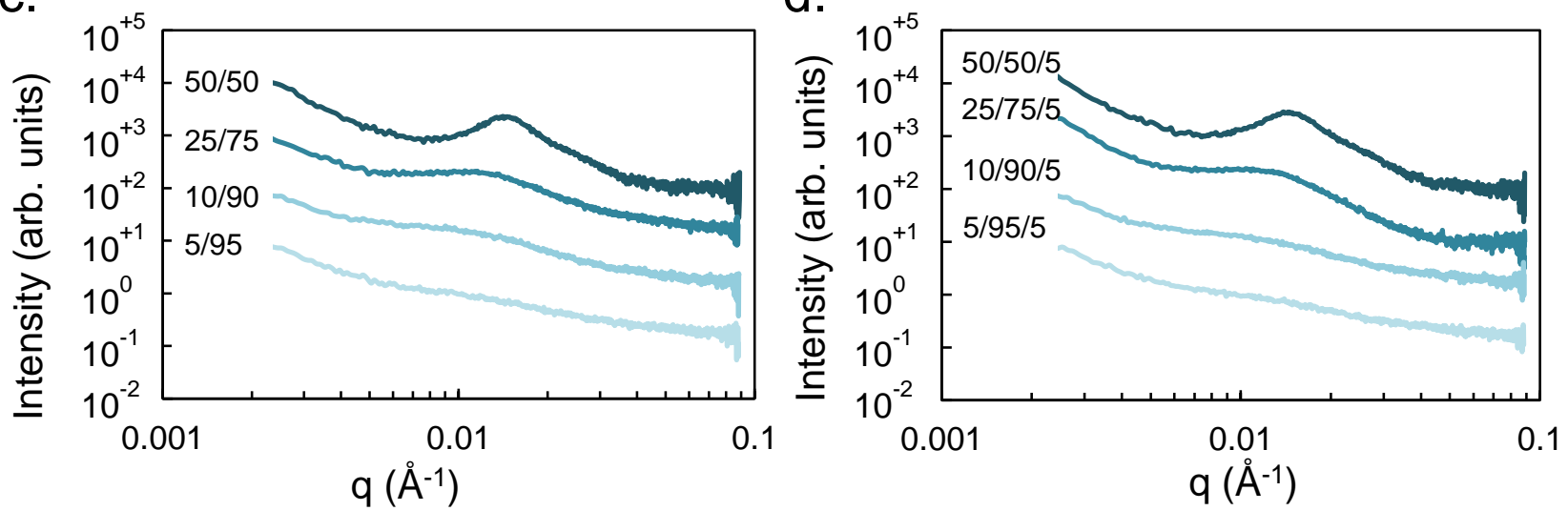

Figure 1. Representative cross-sectional TEM micrographs of ultramicrotomed as-cast (a) binary (PMMANP/SAN) and (b) ternary (PMMA-NP/SAN/PMMA) films as a function of nanoparticle loading showing individually dispersed PMMA-NPs. Image scale bars are $300 \mathrm{~nm}$. Figures 1(c) and (d) show the corresponding 1D SAXS profiles for the binary and ternary PNC films. The profiles are vertically shifted for clarity.

Figures $1 \mathrm{a}$ and $1 \mathrm{~b}$ show representative TEM micrographs of as-cast (i.e. room temperature) binary and ternary PNC films as a function of composition, respectively. The binary PMMA-NP/SAN polymer blend nanocomposites are denoted by $\mathrm{A} / \mathrm{B}$, where $\mathrm{A}$ and $\mathrm{B}$ represent the weight fractions of PMMA-NP and SAN, respectively. The ternary PMMANP/SAN/PMMA PNCs are denoted by $\mathrm{A} / \mathrm{B} / \mathrm{C}$, where $\mathrm{A}$ and $\mathrm{B}$ are the weight fractions of 
PMMA-NP and SAN from the binary PNCs, and $\mathrm{C}$ is the weight fraction of the PMMA compatibilizer with respect to the total amount of polymer (PMMA-NP, PMMA, and SAN). This notation is adopted to more easily compare the two systems. ${ }^{21}$ The images reveal that the films exhibit remarkable dispersion of particles with little to no clustering. The lowest loadings of particles (5/95 and 5/95/5) result in average center-to-center interparticle distances (ID) of ca. 70 $\mathrm{nm}$, as determined through image analysis using ImageJ. The IDs decrease rapidly as the weight percent of NPs increases, resulting in a value of $c a .30 \mathrm{~nm}$ at the highest loadings (50/50 and 50/50/5). Transmission SAXS was utilized as a second method to confirm dispersion and to measure the center-to-center NP correlation lengths. As shown in Figures 1c and 1d, a scattering peak occurs at progressively lower q values as particle loading decreases. The relative magnitude of each peak decreases as loading decreases, consistent with the reduced scatterer populations (scattering at compositions of 5/95 and 5/95/5 was too weak to produce any discernable peak). IDs extracted from hard sphere structure factor fits to the data were $c a .70 \mathrm{~nm}\left(\mathrm{q} \sim 0.009 \dot{\mathrm{A}}^{-1}\right)$ at $10 \mathrm{wt} . \%$ NPs and decreases to $c a .42 \mathrm{~nm}\left(\mathrm{q} \sim 0.015 \dot{\mathrm{A}}^{-1}\right)$ at $50 \mathrm{wt} . \%$. These values are in good agreement with those obtained through autocorrelation functions of each TEM micrograph (Figure S6). For instance, the lowest loadings of particles results in no correlation peak, while the highest loadings (50 wt.\%) exhibits an average center-to-center ID of ca. $45 \mathrm{~nm}$. For NPs that are randomly distributed in the polymer matrix, the theoretical ID is given by ${ }^{44,45}$

$$
\operatorname{ID}(\sigma)=\mathrm{d}_{\mathrm{m}}\left\{\left(\frac{\pi}{6 \varphi_{\mathrm{NP}}}\right)^{\frac{1}{3}}\left[\exp \left(1.5(\ln \sigma)^{2}\right)\right]-\exp \left[0.5(\ln \sigma)^{2}\right]\right\}
$$

where $\mathrm{d}_{\mathrm{m}}$ is the mean diameter $(15.5 \mathrm{~nm}), \varphi_{\mathrm{NP}}$ is the volume fraction of NP cores, and $\sigma$ is the NP polydispersity (Equation S2). The ID measured here is consistent with the theoretical predictions, indicating that the as-cast PNC films are homogeneous and mixing between the grafted NP and matrix polymers is thermodynamically favorable (Figure S7). 


\section{ii.) Phase Separation in Binary and Ternary PNCs}

To probe differences in mixing/demixing behavior of the binary and ternary PNC, phase diagrams are mapped out as a function of temperature and composition. Starting with the well dispersed NP systems at room temperature, the films are annealed between $155^{\circ} \mathrm{C}$ and $195^{\circ} \mathrm{C}$ and analyzed using a combination of cloud point observations, TEM, and SAXS. The phase diagram of the binary PNC serves as a reference system and is determined by monitoring the cloud point transitions of $c a .2 \mu \mathrm{m}$ bulk films (Figure S8). The films transitioned from transparent to opaque with increasing annealing temperature, with a lower critical solution temperature (LCST) of $\approx 160^{\circ} \mathrm{C}$, consistent with PMMA/SAN blends studied by Newby, et al. ${ }^{46}$ This behavior demonstrates that PMMA grafted nanoparticles $\left(\Sigma=0.7 \mathrm{grafts} / \mathrm{nm}^{2}\right)$ in a SAN matrix exhibit enthalpic interactions comparable to the PMMA/SAN blend. Specifically, the large grafting density of PMMA chains shields the NP core such that core-core interactions are effectively screened. The PNC morphology is therefore dictated by the thermodynamic behavior of PMMA and SAN rather than NPs' core-core interactions. While this binary PNC behavior is not new, ${ }^{13,14}$ the effect of adding a third component on phase separation is still unknown. Here, we add the identical polymer as the brush, namely PMMA, at a fixed composition (5 wt.\%), and match their

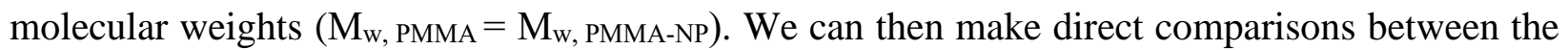
interactions of SAN matrix and grafted PMMA versus free PMMA chains. ${ }^{14}$ Similar to the binary PNC, cloud point measurements (not shown here) were performed on the ternary PNC to determine the phase diagram. Interestingly, cloud point observations suggest a slight increase in miscibility for compositions of 5/95/5 and 10/90/5 where PMMA-NPs are the minority component. 

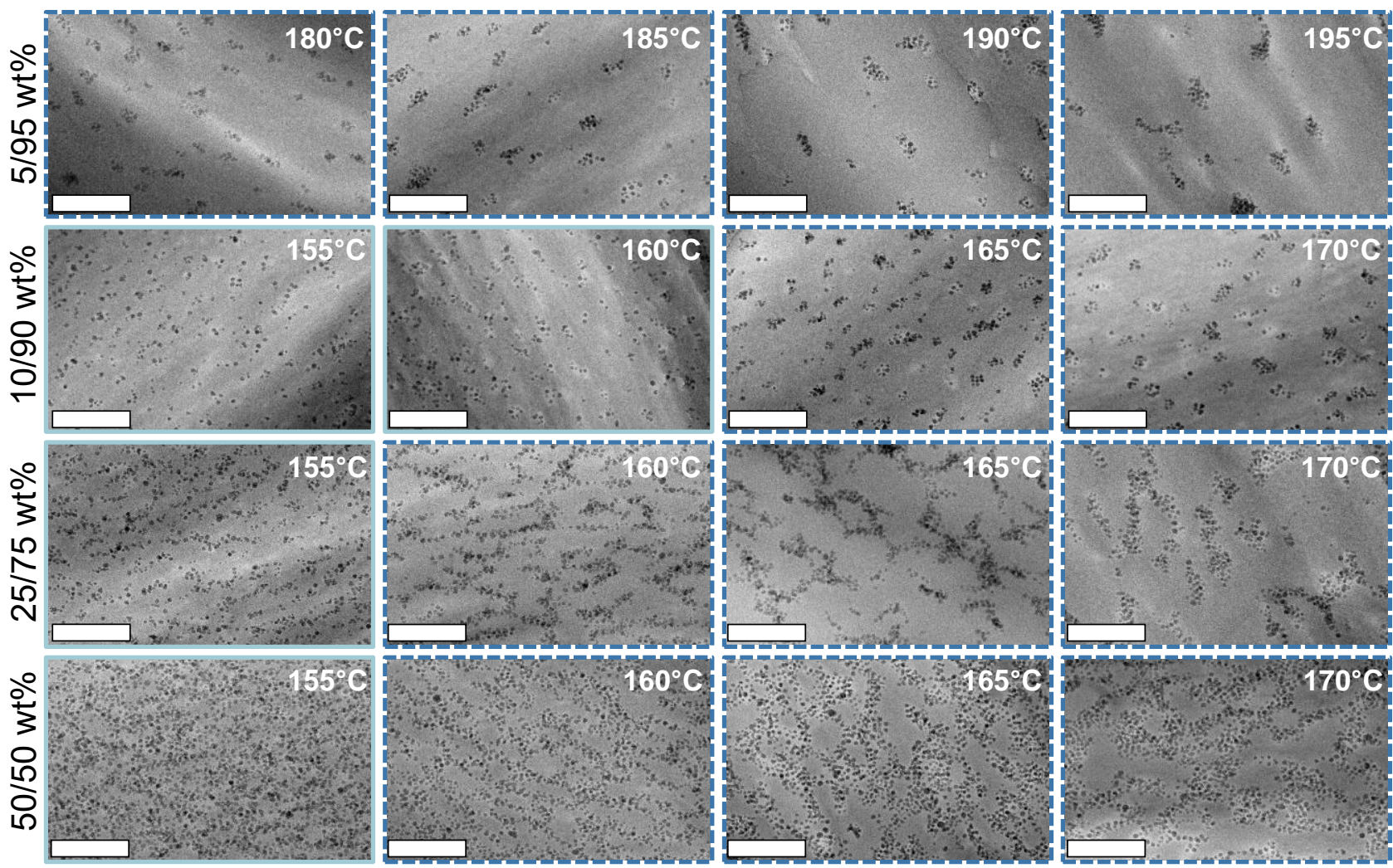

Figure 2. Representative cross-sectional TEM micrographs of ultramicrotomed binary PMMA-NP/SAN $=5 / 95$

(row 1), 10/90 (row 2), 25/75 (row 3), and 50/50 (row 4) films annealed at the indicated temperatures for $24 \mathrm{~h}$.

Border colors denote one (teal, solid) and two (blue, dashed) phase regions. The scale bars are $300 \mathrm{~nm}$.

A more quantitative characterization of PNC phase evolution can be obtained from TEM and SAXS. Figure 2 depicts representative TEM images of binary PNCs at varying compositions: PMMA-NP/SAN $=5 / 95,10 / 90,25 / 75$, and 50/50 after 24 hours of annealing at the respective temperatures. At temperatures below the optically determined phase boundary of $\approx 160^{\circ} \mathrm{C}$, the TEM micrographs reveal that the overall distribution of NPs is homogeneous as shown by the morphologies outlined by the solid teal boxes. Notably, there are several dispersed NP clusters within the one-phase region. This suggests that the potential of mean force between two particles is not purely repulsive and slight aggregation occurs at $\mathrm{T}>\mathrm{T}_{\mathrm{g}}$, similar to what has 
been seen previously. ${ }^{47,48}$ However, as the samples are quenched into the two-phase region, macro-phase separation readily occurs as shown by morphologies outlined by the dashed blue boxes. The degree of phase separation appears to increase with temperature, which is expected based on the increased mobility of the constitutents and deeper quench depth. Interestingly, discrete NP domains are formed for 5/95 and 10/90 off-critical compositions, whereas the 25/75 system qualitatively exhibits string-like and more connected domains. The 50/50 system exhibits a bicontinuous morphology, similar to the previously reported PMMA/SAN blend. ${ }^{46,49}$ Within domains of NP aggregates, the average center-to-center ID is $c a .28 \mathrm{~nm}$ as quantified through image analysis. 50/50 samples were also annealed for $72 \mathrm{~h}$ in order to confirm that the $24 \mathrm{~h}$ annealing times were sufficiently long to reach quasi-equilibrium morphologies, (Figure S9). Specifically, after annealing in the one-phase region, no discernable difference in PNC morphologies is observed at $24 \mathrm{~h}$ and $72 \mathrm{~h}$. Likewise, when annealed in the two-phase region, the aggregate domain size only slightly increases after $72 \mathrm{~h}$ compared to $24 \mathrm{~h}$. This comparison indicates that $24 \mathrm{~h}$ is sufficient to distinguish between one-phase and two-phase morphologies in the binary and ternary PNCs.

Figure 3 shows cross-sectional TEM micrographs for ternary compositions analogous to the binary systems shown in Figure 2 after $24 \mathrm{~h}$ of annealing at the indicated temperatures. For $5 / 95 / 5$ films, particles remain dispersed at temperatures up to $180^{\circ} \mathrm{C}$. At temperatures of $185^{\circ} \mathrm{C}$ and higher, the uniform distribution of NPs becomes disrupted and the NPs form discrete aggregates that increase in size as temperature increases. Similarly, 10/90/5 films exhibit macrophase separation into discrete domains at temperatures of $170^{\circ} \mathrm{C}$. While comparable, the reduced domain size for the 10/90/5 composite compared to the 5/95/5 is attributed to the smaller thermodynamic driving force and slower diffusivity at lower temperatures. The phase separation 
evolution of PNCs near the phase separation temperature $\left(\approx 160^{\circ} \mathrm{C}\right)$ and at intermediate compositions (i.e., 25/75/5 and 50/50/5) show a transition from dispersed to aggregated, forming
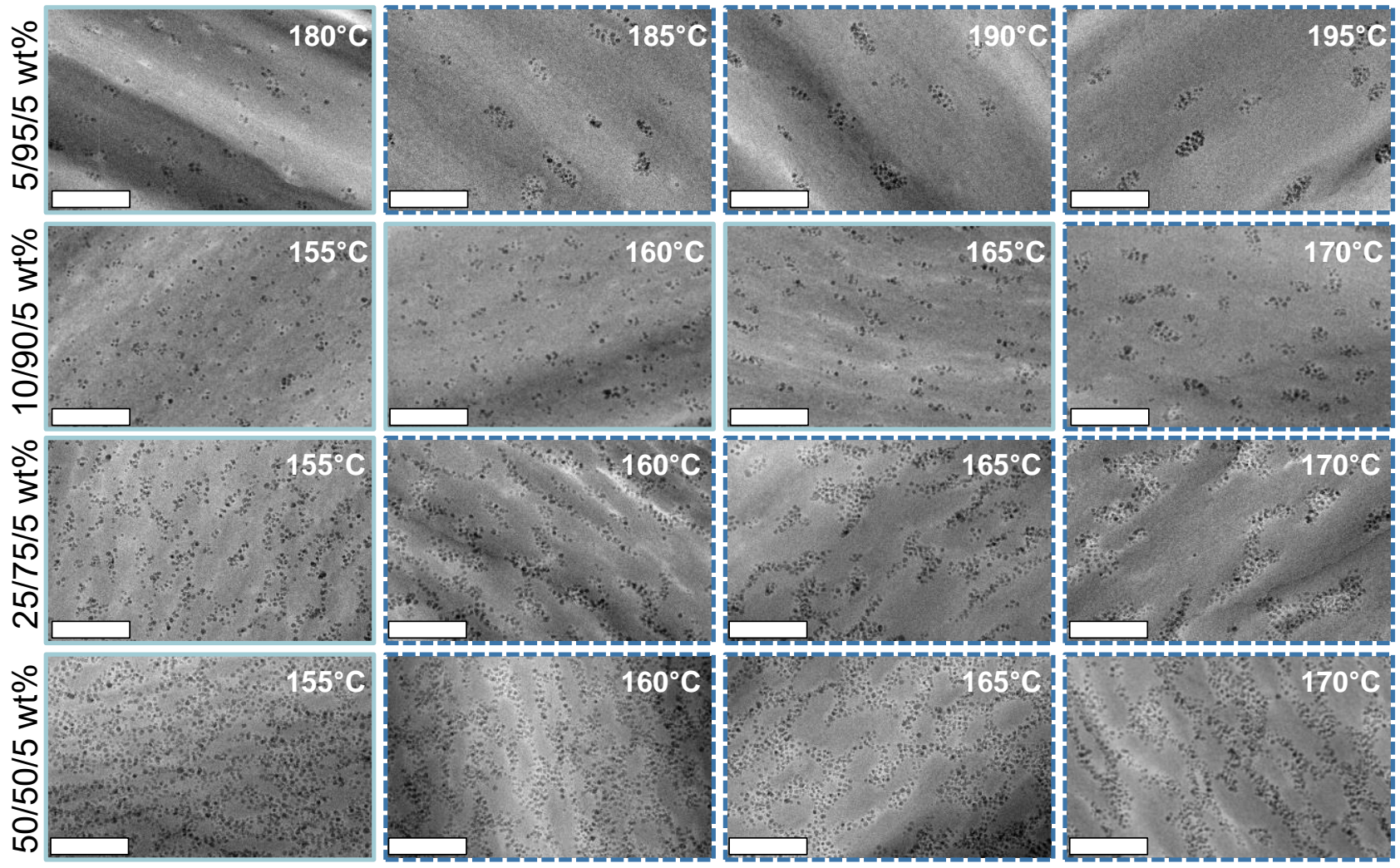

Figure 3. Representative cross-sectional TEM micrographs of ultramicrotomed ternary PMMA-NP/SAN/PMMA

$=5 / 95 / 5$ (row 1), 10/90/5 (row 2), 25/75/5 (row 3), and 50/50/5 (row 4) films annealed at the indicated temperatures for $24 \mathrm{~h}$. Border colors denote one (teal, solid) and two (blue, dashed) phase regions. The scale bars are $300 \mathrm{~nm}$.

string-like and bicontinuous morphologies, respectively.

While these trends for ternary composites are similar to the binary composite, several interesting features emerge. Specially, off-critical compositions of 5/95/5 (180 $\left.{ }^{\circ} \mathrm{C}\right)$ and 10/90/5 $\left(165^{\circ} \mathrm{C}\right)$ reveal, on average, uniformly distributed particles and clusters of much smaller size compared to their binary counterpart. At $180^{\circ} \mathrm{C}, 5 / 95 / 5$ ternary composites exhibit significantly less aggregation compared to the binary analogue (Figure S10). This aggregation suppression is 
also observed in the 10/90/5 case, but less pronounced. Compositions approaching the critical composition show no appreciable difference in miscibility upon addition of linear PMMA,

a.

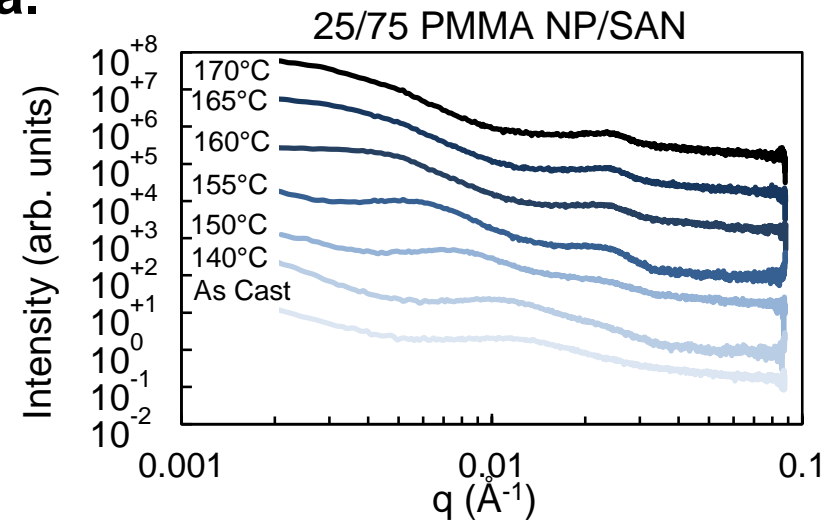

c.

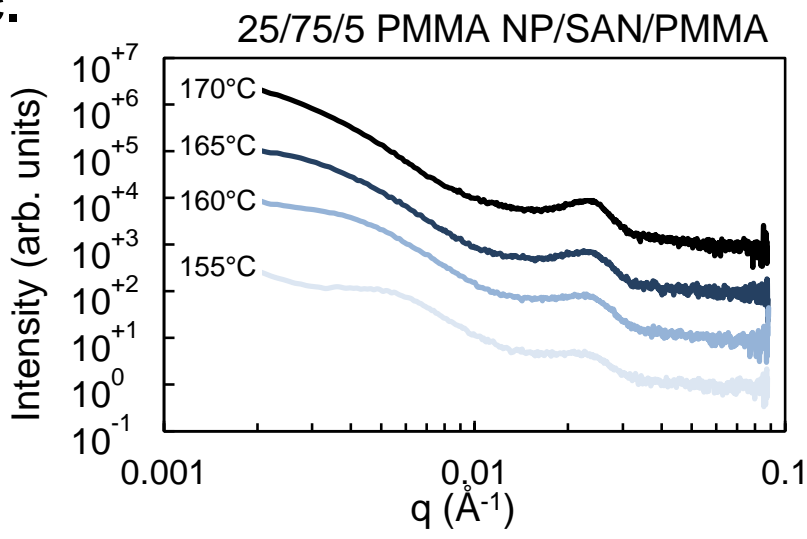

b.

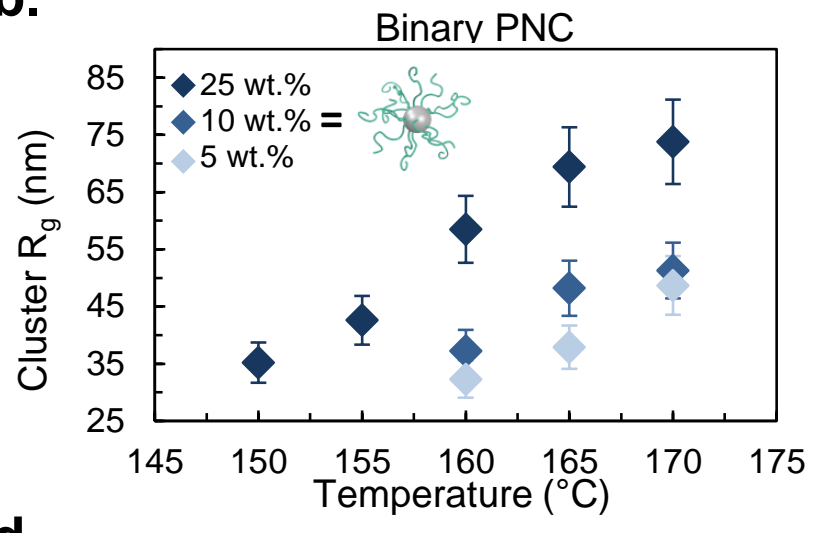

d.

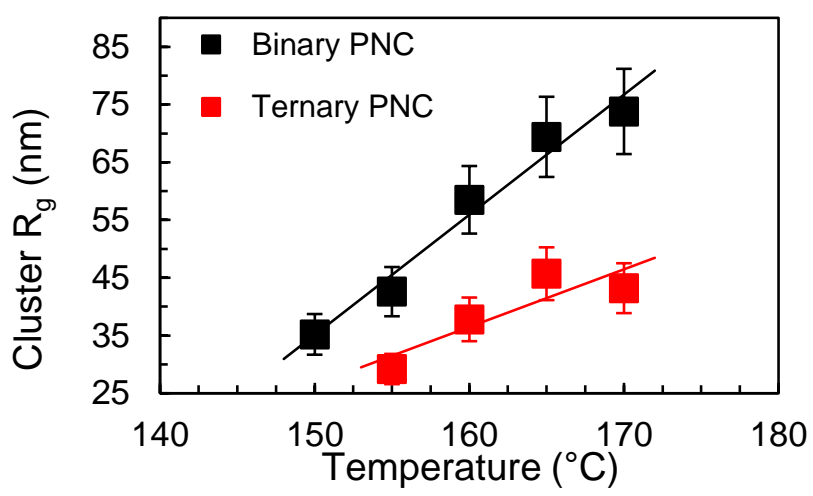

Figure 4. (a) 1D SAXS profiles of 25/75 wt. $\%$ binary PMMA-NP/SAN films annealed at the indicated temperatures for $24 \mathrm{~h}$. Figure $4 \mathrm{~b}$ shows the corresponding average nanoparticle cluster radius of gyration as a function of temperature obtained through SAXS modeling. Figure 4c shows 1D SAXS profiles for the 25/75/5 wt.\% ternary PNC under identical annealing conditions to those in (a). A direct comparison of the average aggregate size as a function of temperature for (a) and (c) is shown in Figure 4d. The 1D SAXS profiles are vertically shifted to avoid overlap and more clearly track evolutions in scattering peaks.

similar to what was observed in the cloud point transitions.

We performed SAXS measurements on the annealed PNCs in parallel with TEM to further quantify the length scales of phase separation as a function of temperature and NP loading. Figure 4a shows the 1D scattering intensity as a function of wavevector for a 
representative 25/75 wt.\% binary PNC (data for other compositions are in Supporting Information). The background-subtracted 1-dimensional scattering intensities were modeled using two scattering contributions to represent isolated/paired NPs and aggregated NP clusters (see Supporting Information). We first consider the scattering profile for the as-cast samples, which shows a single, weak scattering feature near $\mathrm{q} \sim 0.0123 \AA^{-1}$. This represents the average center-to-center interparticle distance $(51 \mathrm{~nm})$ which is greater than the diameter of a single grafted NP. This observation is consistent with well-dispersed NP cores, in agreement with TEM images in Figure 1. However, after annealing, we observe the emergence of two separate scattering features; one at higher q, and one at lower q. The peak position of the higher q feature remains relatively unchanged at higher temperatures, while the lower q feature increases in intensity while also moving to even smaller values. Modeling of the data indicates that the higher q feature represents the average center-to-center interparticle distance, which initially (slightly) decreases with increasing temperature, and eventually becomes well defined as the PMMA-NPs aggregate at elevated temperatures. The lower q feature represents the scattering between the small nanoparticle clusters as seen in Figures 2 and 3, which continue to grow at elevated temperatures as smaller clusters coalesce. Both of these observations indicate a phase transition between $\approx 150-160^{\circ} \mathrm{C}$, commensurate with observations from TEM. The scattering feature representing the aggregated NPs can be used to determine the average size of a single 'cluster' of NPs. For the binary composite at 5, 10 and 25 wt. $\%$, Figure $4 \mathrm{~b}$ shows how the average cluster radius of gyration $\left(\mathrm{R}_{\mathrm{g}}\right)$ increases as temperature increases, consistent with an increase in the thermodynamic driving force for phase separation. For example, at $25 \mathrm{wt} . \%, \mathrm{Rg}_{\mathrm{g}}$ increases by more than a factor of two as temperature increases from $150^{\circ} \mathrm{C}$ to $170^{\circ} \mathrm{C}$. There is also a significant increase in the cluster size as the NP loading increases from $10 \mathrm{wt} . \%$ to 25 
wt.\%, consistent with a smaller as-cast interparticle distance at higher loading (cf. Figure S7). Namely, because NPs are initially closer to one another at higher loadings, larger aggregates are easier to form for the same annealing times.

It is important to note that even at temperatures below the $\operatorname{LCST}\left(160^{\circ} \mathrm{C}\right)$, a shoulder can be observed in the scattering intensity (Figure 4a) at q values close to the aggregate ID. Based on the TEM micrographs in Figure 2, we interpret this as the formation of small NP clusters when LCST $>\mathrm{T}>\mathrm{T}_{\mathrm{g}}$ even though macro-phase separation is not thermodynamically favorable. Annealing the samples above their glass transition allows the NPs to diffuse more readily, leading to the formation of small clusters due to the short-range attractive forces between the particles noted earlier. Noticeably, the measured ID $(28 \mathrm{~nm})$ for the center-to-center interparticle distance is slightly less than the diameter of the PMMA-NP, suggesting that PMMA brushes are weakly interpenetrating with one another at elevated temperatures.

Figure 4c shows scattering data for the PMMA-NP/SAN/PMMA ternary composite at identical NP loadings as the binary system shown in Figure 4a. Modeling of the data reveals two notable differences between the binary and ternary systems. First, the high- q scattering feature is at slightly smaller q-values in the ternary PNC than in the binary one at $170^{\circ} \mathrm{C}\left(0.024 \AA^{-1}\right.$ vs. $0.026 \AA^{-1}$, respectively). This is attributed to less PMMA brush interdigitation and a lower degree of brush collapse, indicative of a slightly more miscible PNC. Second, the average NP aggregate size is smaller in the ternary composite than in the binary case. This reduction in cluster size is substantially more than what one could surmise from a simple dilution argument through the addition of the ternary component. Figure $4 \mathrm{~d}$ compares the aggregate size as a function of temperature for the binary (black squares) and ternary (red squares) systems. Here, it is worth noting that in addition to smaller aggregate sizes, the difference between aggregate sizes 
of the binary and ternary PNCs increases as the temperature increases. The addition of only 5 wt.\% PMMA homopolymer decreases the average aggregate size by as much as a factor of two at $170^{\circ} \mathrm{C}$, indicating a suppression in aggregation and an increase in PNC miscibility.

Figure 5 shows a phase diagram for both binary and ternary composites constructed by compiling cloud point, TEM, and SAXS results. Near the critical temperature, the binary phase diagram is relatively flat between $20 \mathrm{wt} . \%$ and $50 \mathrm{wt} . \%$ PMMA-NP and increases sharply below 10 wt. $\%$ and above 63 wt.\%. Accounting for the polydispersity of SAN, this behavior is consistent with other blends containing a polydisperse component. ${ }^{50}$ From the observed phase separation behavior, the

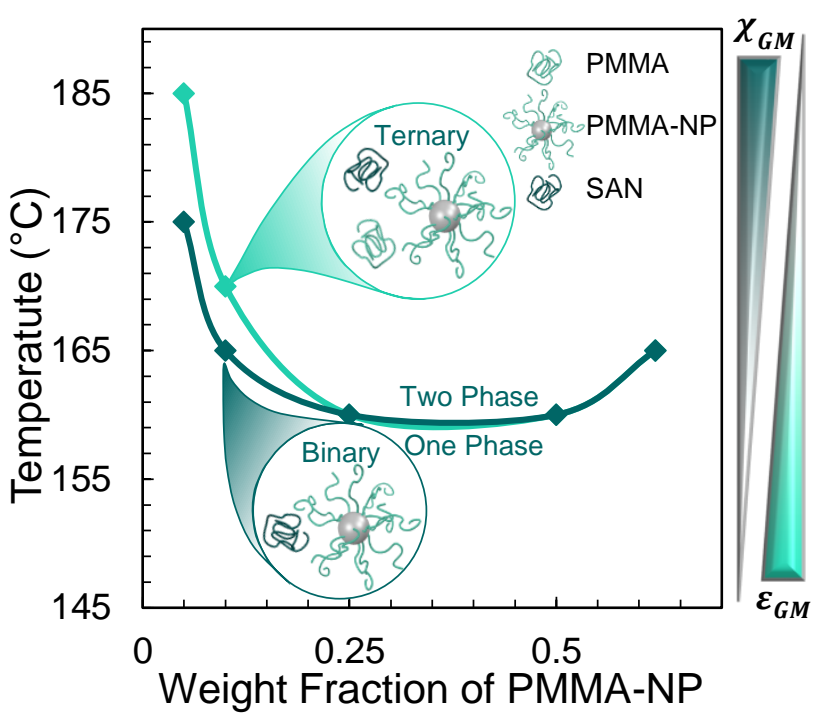

Figure 5. Phase diagrams of binary PMMA-NP/SAN and ternary PMMA-NP/SAN/PMMA nanocomposites. The ternary composites exhibit increased miscibility in off-critical compositions. The solid lines have been drawn to guide the eye.

addition of linear PMMA increases the miscibility of the binary PMMA-NP/SAN system at the most off-critical compositions (i.e., 5/95/5) by as much as $10^{\circ} \mathrm{C}$. To begin to understand this, we recall the work presented by Schmitt et al., who studied a binary system of silica particles grafted with polystyrene (PS) and PMMA ligands with an upper critical solution temperature (UCST). They demonstrated that the specific ligand interactions in mixtures of these polymer-grafted nanoparticles imparts similar phase behavior as in analogous linear polymer blends, similar to our findings for PMMA/SAN systems. ${ }^{13}$ While general trends in phase behavior between the two systems were alike, distinct differences were recognized. Specifically, the binary PNC 
demonstrated increased miscibility and slower kinetics of domain growth as compared to the neat polymer blend. The apparent increase in miscibility was rationalized as a consequence of a reduced thermodynamic driving force for phase separation attributed to fewer unfavorable segmental interactions between PMMA-PS ligands. Additionally, the presence of NP cores resulted in a smaller number density of segmental contacts. ${ }^{13}$ Here, it is important to reiterate that these segmental interactions are unfavorable due to the systems UCST behavior. Therefore, the UCST work by Schmitt and co-workers is consistent with the presented LCST work thus far, with our results demonstrating that the addition of linear PMMA reduces aggregation of PMMANPs and increases miscibility. We attribute this phenomena to an interfacial compatibilization between the SAN matrix and grafted PMMA brush due to localization of the free PMMA chains. The addition of linear PMMA increases the number of favorable segmental contacts between each chemical species and therefore reduces the thermodynamic driving force for phase separation (parsing of the specific enthalpic and entropic contributions to this will be discussed later). Importantly, this miscibility increase was only observed for 5/95/5 and 10/90/5 off-critical compositions. These experimental observations will be supported by PRISM calculations and MD simulations (vide infra).

The improved miscibility from homopolymer compatibilization can be understood from a simple geometric model that considers the interaction between the free and grafted PMMA chains. Under the assumption that the free PMMA chains are Gaussian coils with a pervaded volume described as spheres, the number of chains needed to cover the surface area of a PMMANP can be directly calculated. ${ }^{51}$ As shown in Figure 6, two regimes are considered: (1) PMMA chains wetting the PMMA brush (wet brush state) and (2) PMMA excluded from the PMMA brush layer (dry brush state). For the wet brush case, $c a$. 650 homopolymer chains are needed to 
completely cover a PMMA-NP/matrix interface, whereas $c a$. 740 chains are needed for the dry brush state. The total number of available PMMA homopolymer chains for compatibilization at a constant $5 \mathrm{wt} . \%$ loading can be calculated. Under wet brush conditions, the ratio of required to available PMMA chains for the 5/95/5 composite is approximately $1: 1$. As the composition of the films increases to higher loadings of PMMA-NPs, the ratio decreases to 2:1, 4.5:1, and 9:1 for $10 / 90 / 5,25 / 75 / 5$, and 50/50/5 films, respectively. While similar, the ratios derived for the dry brush regime are smaller given that more PMMA homopolymer is needed to shield the NP surface. Due to the small amount of PMMA-NPs in 5/95/5 films, the proposed scaling model suggests that a 5 wt.\% addition of PMMA is sufficient to compatibilize the PMMA-NP/SAN system. Specifically, at low loadings of PMMA-NPs, there is sufficient PMMA homopolymer to completely wet the PMMA brush layer, and the resultant phase behavior is dictated by the PMMA homopolymer and SAN interactions. However, as the concentration of NPs increases, 5 wt.\% of PMMA becomes insufficient to completely shield the PMMA brush from the SAN matrix, and the phase behavior is dictated by the brush/matrix interactions as demonstrated by the aforementioned ratios. This behavior is captured by our experimental findings shown in Figure 5 where the highest miscibility increase occurred in 5/95/5 films, and progressively decreased with particle loading. 
a.

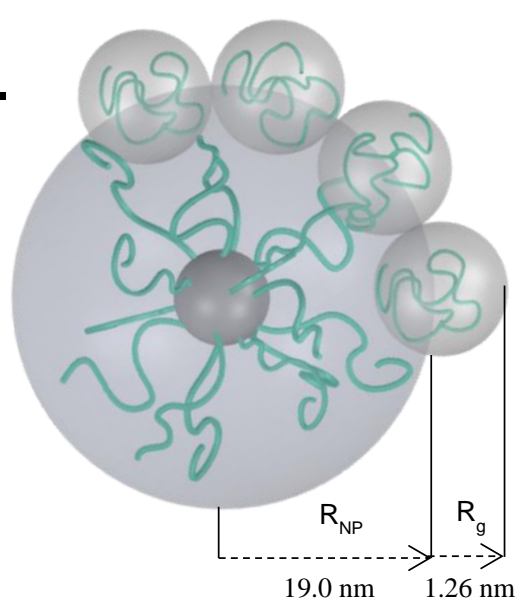

b.

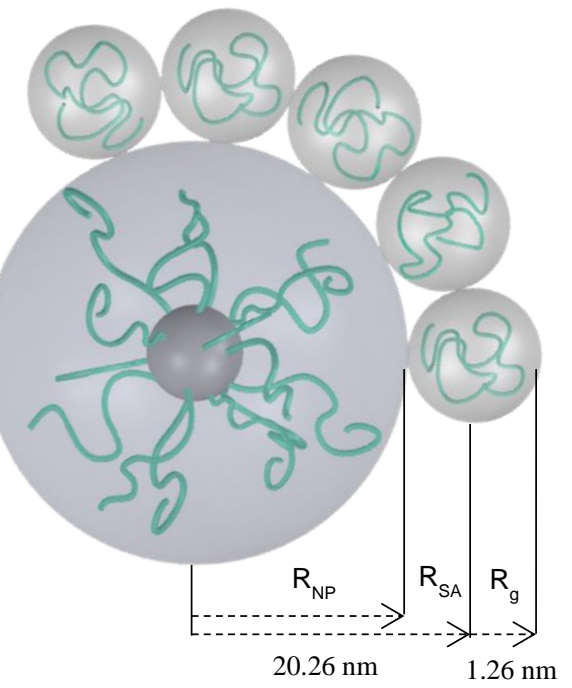

Figure 6. This cartoon illustrates two possible scenarios of linear PMMA covering the surface the PMMA-NPs. Case one (a) assumes that linear PMMA chains wet the PMMA-NP brush layer. Case two (b) adopts both PMMANPs and linear PMMA as hard spheres, resulting in complete exclusion of PMMA chains within the brush corona. 


\section{iii.) Comparing Computations and Experiments}
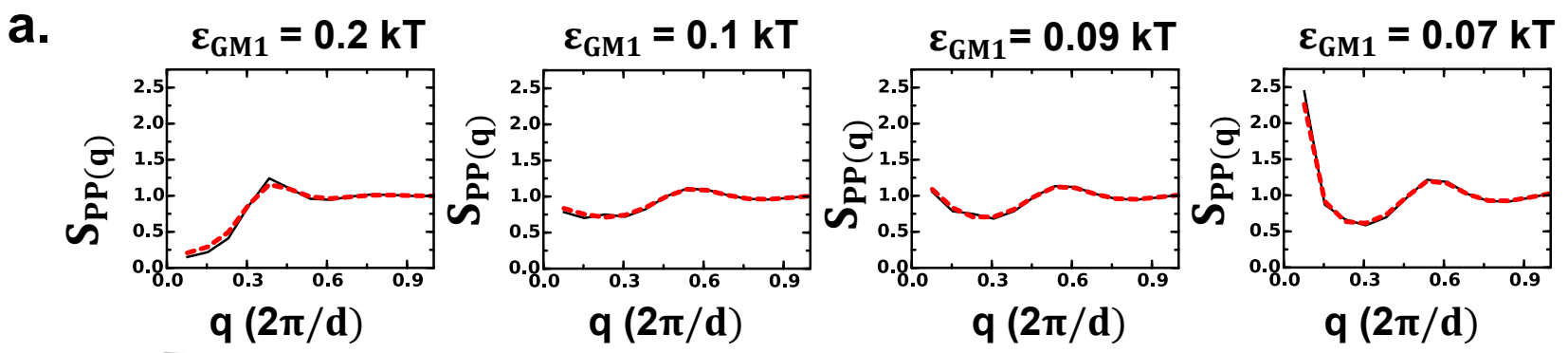

\section{$\varepsilon_{G M 1}$}

b.

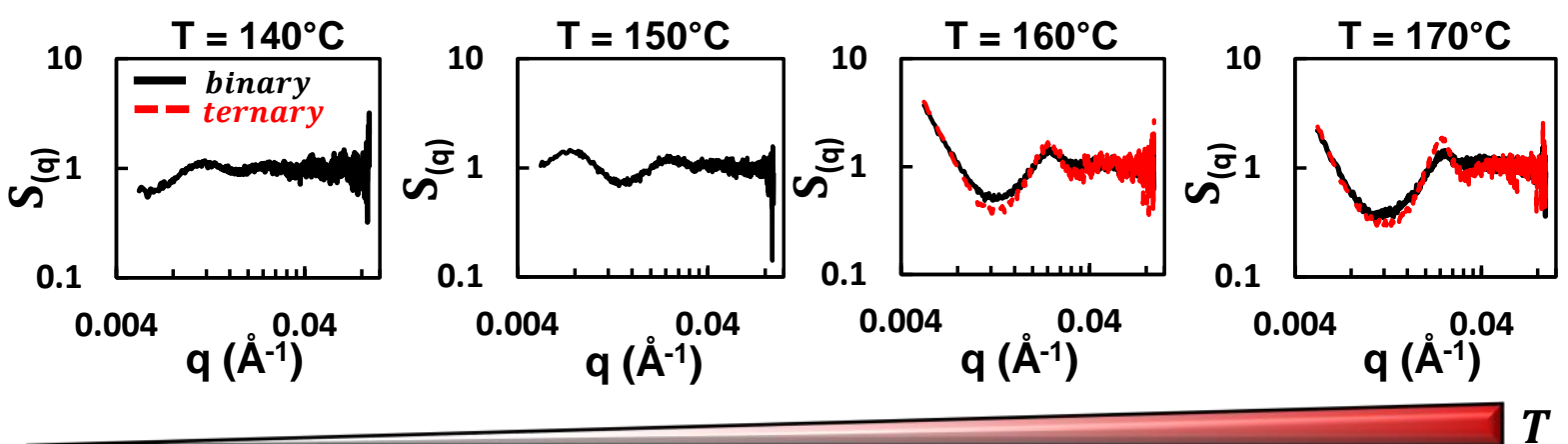

Figure 7. (a) Particle-particle structure factors, $\mathrm{S}_{\mathrm{PP}}(\mathrm{q})$, obtained using PRISM theory for PNCs with $\phi_{\mathrm{PGP}}=$ $0.205, \varepsilon_{\mathrm{GG}}=\varepsilon_{\mathrm{M} 1 \mathrm{M} 1}=0.1 \mathrm{kT}, \mathrm{D}_{\mathrm{P}}=5 \mathrm{~d}, \mathrm{~N}_{\mathrm{G}}=15, \mathrm{~N}_{\mathrm{M} 1}=75, \mathrm{~N}_{\mathrm{M} 2}=15, \Sigma=0.76$ chains $/ \mathrm{d}^{2}$ and (left to right) $\varepsilon_{\mathrm{GM} 1}=$ $0.2 \mathrm{kT}, \varepsilon_{\mathrm{GM} 1}=0.1 \mathrm{kT}, \varepsilon_{\mathrm{GM} 1}=0.09 \mathrm{kT}$ and $\varepsilon_{\mathrm{GM} 1}=0.07 \mathrm{kT}$. The binary PNC case with $\phi_{\mathrm{M} 2}=0$ is shown as a black line and the ternary case with $\phi_{\mathrm{M} 2}=0.043(5 \mathrm{wt} \%$ ) is shown as a red dashed line. (b) Corresponding particle-particle structure factors, S(q), obtained using SAXS for 25/75 wt.\% PNCs.

The compatibilization effect of the added PMMA chains in the ternary PNCs can be systematically investigated using molecular dynamics simulations and polymer reference interaction site model (PRISM) theory serving as computational comparison to the experimental results. As a reminder, in the model the notation graft $(\mathrm{G})$ and matrix_2 (M2) refer to grafted PMMA and free PMMA matrix chains, and matrix_1 (M1) refers to the SAN matrix chains in experiments. The choice of the graft-matrix_1 $\left(\varepsilon_{\mathrm{GM} 1}\right)$, graft-graft $\left(\varepsilon_{\mathrm{GG}}\right)$, and matrix_1-matrix_1 ( $\left.\varepsilon_{\mathrm{M} 1 \mathrm{M} 1}\right)$ attraction strengths at a constant $\varepsilon_{\mathrm{GG}}$ and $\varepsilon_{\mathrm{M} 1 \mathrm{M} 1}$ of $0.1 \mathrm{kT}$ in our model dictates whether 
the modeled PNC exhibits net favorable interactions (i.e. below the LCST), effectively athermal interactions, or net unfavorable interactions (i.e. above the LCST). Figure 7a shows a series of particle-particle structure factors calculated using PRISM theory for PNCs with decreasing values of $\varepsilon_{\mathrm{GM} 1}$; the corresponding structure factors from experiments at increasing temperatures are also shown in Figure $7 \mathrm{~b}$. The $\mathrm{SPP}_{\mathrm{P}}(\mathrm{q} \rightarrow 0)$ for binary (black solid lines) and ternary (red dashed lines) composites at $\varepsilon_{\mathrm{GM} 1}=0.2 \mathrm{kT}$, corresponding to low temperatures in experiments, shows no upturn, suggesting absence of macrophase separation and confirming that the nanoparticles remain relatively well-dispersed. This is in agreement with the experimentally obtained structure factors for systems below the LCST (Figure $7 \mathrm{~b}$ ). For the binary and ternary composites at $\varepsilon_{\mathrm{GM} 1}=$ $0.1 \mathrm{kT}$, there is a distinct increase in both the value of $\mathrm{SPP}_{\mathrm{P}}(\mathrm{q} \rightarrow 0)$ as well as the slope of $\mathrm{SPP}_{\mathrm{P}}(\mathrm{q})$ at low q values as compared to the PNCs with $\varepsilon_{\mathrm{GM} 1}=0.2 \mathrm{kT}$. Furthermore, both PNCs with $\varepsilon_{\mathrm{GM} 1}$ $=0.09 \mathrm{kT}$ and $0.07 \mathrm{kT}$ show an upturn when $\operatorname{SPP}(q \rightarrow 0)$, which is consistent with the shapes of the structure factors obtained from experiments performed at higher temperatures where phase separation occurs. These results confirm that decreasing values of $\varepsilon_{\mathrm{GM} 1}$ at constant $\varepsilon_{\mathrm{GG}}$ and $\varepsilon_{\mathrm{M} 1 \mathrm{M} 1}$ in the $\mathrm{CG}$ model qualitatively captures the behavior in experiments with increasing temperature. This CG model can thus be used in MD simulations and PRISM theory to probe and explain the hypothesized compatibilization observed experimentally in the ternary PNCs. Whereas the amount of PMMA in ternary PNCs is kept at $5 \mathrm{wt} . \%$ for the experimental systems, MD simulations and PRISM theory allow us to systematically investigate the effect of increasing the PMMA matrix volume fraction, $\phi_{\mathrm{M} 2}$, on the structure of the grafted chains and the bulk PNC morphology. In both theory and simulations, the PMMA matrix volume fractions of $\phi_{\mathrm{M} 2}=0$ and $\phi_{\mathrm{M} 2}=0.043$ correspond to the binary and ternary (5 wt.\% PMMA) PNC cases in experiments, respectively. Additionally, we also show here the results for a higher $\phi_{\mathrm{M} 2}$ case of 
0.1 and the limiting case of $\phi_{\mathrm{M} 2} \approx 1$ (binary PNC with PMMA grafts and chain length matched PMMA matrix) to elucidate the effect of increasing PMMA volume fraction on structure. In the SI we present the results for ternary PNCs for all $\phi_{\mathrm{M} 2}$ ranging from 0 to 1 .

First, we use MD simulations to see if we observe compatibilization by quantifying the grafted layer structure and the extent of interpenetration of the matrix_1 and matrix_2 chains into the grafted layer. In binary PNCs (with $\phi_{\mathrm{M} 2}=0$ ) when $\varepsilon_{\mathrm{GM} 1}=0.2 \mathrm{kT}$, the net interactions between the graft and matrix_1 (SAN) chains are favorable. However, the matrix_1 chain length is much greater than the graft chain length (i.e., $\mathrm{N}_{\mathrm{M} 1}=75>\mathrm{N}_{\mathrm{G}}=15$ ), which leads to entropic driving forces disfavoring graft-matrix_1 interpenetration due to a large loss in conformational entropy of long matrix chains upon wetting the grafted layer. In ternary PNCs with graft and matrix_2 chains being chemically identical and equal in lengths (i.e., $\mathrm{N}_{\mathrm{M} 2}=\mathrm{N}_{\mathrm{G}}=15$ ), the shorter matrix_2 (PMMA) chains have a higher entropic driving force to wet the grafted layer than the enthalpically favored longer matrix_1 (SAN) chains. Thus, it is non-intuitive as to which of these two competing factors dominate. Using concentration profiles of the graft, matrix_1 and matrix_2 chains calculated in MD simulations, we can understand how these two competing forces dictate compatibilization at different $\varepsilon_{\mathrm{GM} 1}$. 

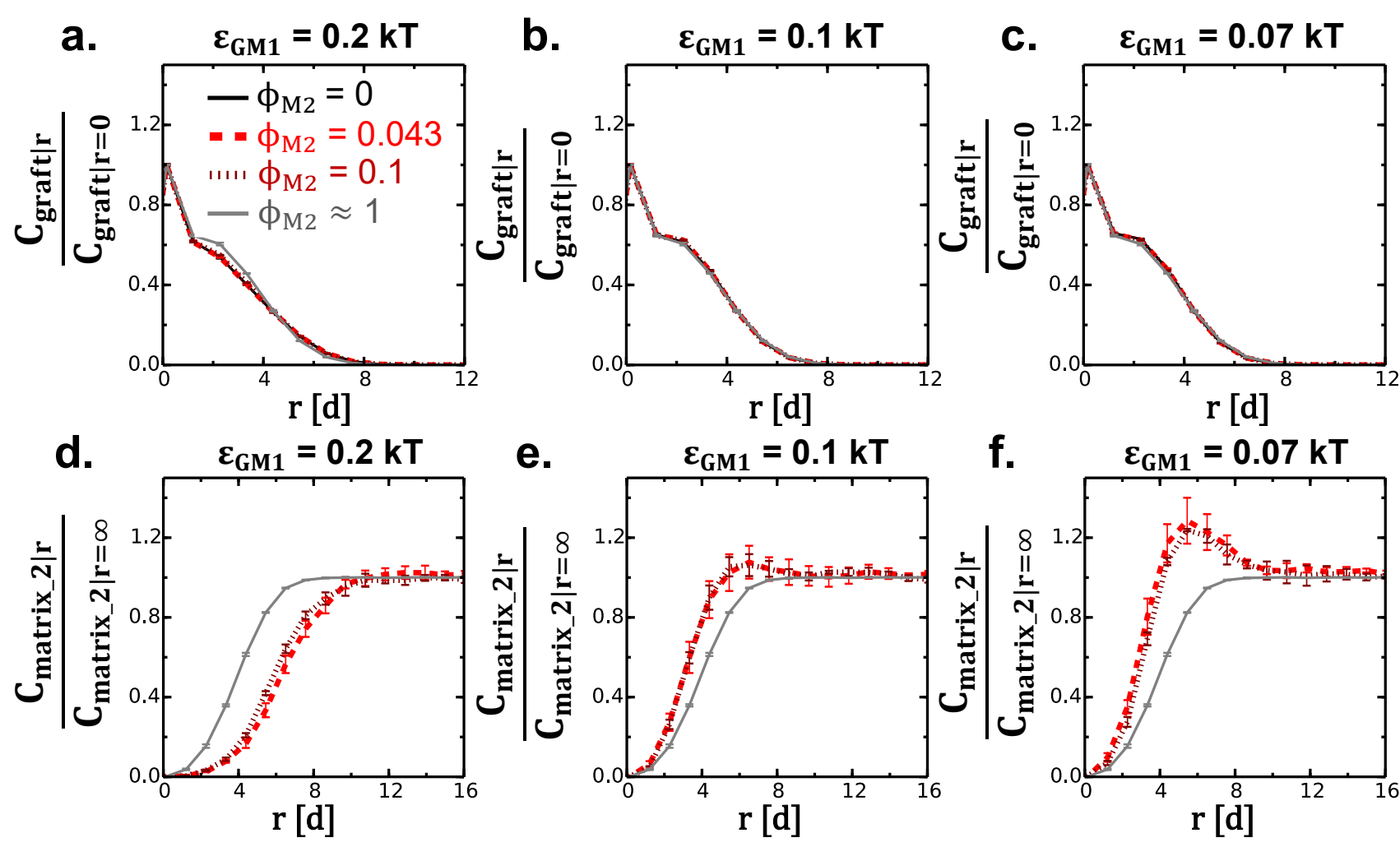

Figure 8. Concentration profiles for graft, $\mathrm{G}(\mathrm{a}, \mathrm{b}$, and c) and matrix 2 , M2 (d, e, and f) beads as a function of distance from the particle surface, $\mathrm{r}[$ in units of $\mathrm{d}]$ using MD simulations for PNCs with $\varepsilon_{\mathrm{GM} 1}=0.2 \mathrm{kT}$ (a, $\mathrm{d}), \varepsilon_{\mathrm{GM} 1}=0.1 \mathrm{kT}(\mathrm{b}, \mathrm{e})$ and $\varepsilon_{\mathrm{GM} 1}=0.07 \mathrm{kT}(\mathrm{c}, \mathrm{f})$. Colored lines represent varying volume fractions of matrix $\_2$ chains with $\phi_{\mathrm{M} 2}=0$ (black line), $\phi_{\mathrm{M} 2}=0.043$ (red dashed line), $\phi_{\mathrm{M} 2}=0.1$ (dark red dotted line) and $\phi_{\mathrm{M} 2} \approx 1$ (gray line). The legend in part (a) applies to all parts in this figure. These results are for $\varepsilon_{\mathrm{GG}}=$ $\varepsilon_{\mathrm{M} 1 \mathrm{M} 1}=0.1 \mathrm{kT}, \mathrm{D}_{\mathrm{P}}=5 \mathrm{~d}, \mathrm{~N}_{\mathrm{G}}=15, \mathrm{~N}_{\mathrm{M} 1}=75, \mathrm{~N}_{\mathrm{M} 2}=15, \Sigma=0.76$ chains $/ \mathrm{d}^{2}$ and $\phi_{\mathrm{PGP}}=0.01$. The error bars have been obtained from block averages of 4 blocks of 50 configurations each, sampled in a simulation run.

Figure 8 shows normalized concentration profiles of the graft (PMMA) and matrix_2 (PMMA) polymers as a function of radial distance from the NP surface for decreasing values of $\varepsilon_{\mathrm{GM} 1}$. At the highest $\varepsilon_{\mathrm{GM} 1}$ value (low temperature) (Figure 8a) the concentration of graft chains in both binary and ternary PNCs with $\phi_{\mathrm{M} 2} \leq 0.1$ are similar. This suggests that adding low amounts of PMMA (matrix_2) does not alter the spatial arrangement of graft polymers around the particle 
when $\varepsilon_{\mathrm{GM} 1}$ is high and $\phi_{\mathrm{M} 2}$ is low. However, when $\phi_{\mathrm{M} 2}=1$, the concentration of graft polymer at $\mathrm{r} \sim 1 d-4 d$ is slightly higher than in PNCs with $\phi_{\mathrm{M} 2}=0-0.1$. Furthermore, at this high $\varepsilon_{\mathrm{GM} 1}$ value (low temperature), the brush height at $\phi_{\mathrm{M} 2}=1$ is smaller than that at lower values of $\phi_{\mathrm{M} 2}$ (Figure S12a). This is because at low $\phi_{\mathrm{M} 2}$, the enthalpic driving forces between the SAN matrix and PMMA graft in PNCs extend the graft polymer more than the favorable entropic driving forces in PNCs with shorter PMMA matrix chains at $\phi_{\mathrm{M} 2} \approx 1$. As $\phi_{\mathrm{M} 2}$ increases we do not see a significant difference in matrix_1 bead concentration profiles (Figure S13). The concentration profiles of matrix_2 beads (Figure $8 \mathrm{~d}$ ) show that increasing $\phi_{\mathrm{M} 2}$ from values of 0.043 to the limiting case of $\phi_{\mathrm{M} 2} \approx 1$ (gray line) leads to increased penetration of the matrix_2 chains into the grafted layer. This is because at $\varepsilon_{\mathrm{GM} 1}=0.2 \mathrm{kT}$ and at low $\phi_{\mathrm{M} 2}$ values, the favorable interactions between SAN polymer (matrix_1) and PMMA grafts (graft) compete with the entropically driven penetration of short PMMA matrix chains (matrix_2) to wet the grafted layer. Simulations of PNCs with additional $\phi_{\mathrm{M} 2}$ values between 0 and 1 (Figure S14d) show that the penetration of matrix_2 chains into the grafted layer progressively increases with increase in $\phi_{\mathrm{M} 2}$ at this $\varepsilon_{\mathrm{GM} 1}$ value.

As $\varepsilon_{\mathrm{GM} 1}$ decreases to $\varepsilon_{\mathrm{GM} 1}=0.1 \mathrm{kT}$ (Figure $8 \mathrm{~b}$ and $8 \mathrm{e}$ ), both the graft-matrix $\_1$ and graftmatrix_2 interactions become comparable, and the entropic driving forces cause matrix_2 chains to penetrate the grafted layer more than the long matrix_1 (SAN) chains. ${ }^{52}$ Comparison of the matrix_2 concentration profiles in ternary PNCs confirms this higher interpenetration at low values of $\varepsilon_{\mathrm{GM}}$. In Figure 8e, the matrix_2 chain concentration at the end of the grafted layer is slightly greater than that of the bulk. In addition, the concentration of the matrix_ 2 chain is greater than the binary PNC case when $\phi_{\mathrm{M} 2} \approx 1$. This suggests that the free PMMA chains are localizing at the PMMA-NP/SAN interface within the ternary PNCs. This effect is further 
amplified at $\varepsilon_{\mathrm{GM} 1}=0.07 \mathrm{kT}$ (Figure 8f) where the shorter and athermal matrix_2 chains have a higher concentration near the grafted layer surface because matrix_1 (SAN) chains are not only longer than graft chains (making it entropically unfavorable to interpenetrate the graft layer), but also interact unfavorably with graft chains. The presence of this increased concentration of matrix_2 near the grafted layer only at low values of $\varepsilon_{\mathrm{GM} 1}$ (high temperature in experiments) agrees with increasing compatibilization seen in experiments (see Figure 4d). Furthermore, Figure 8f and Figure S14f show that this compatibilization effect in ternary PNCs is better at $\phi_{\mathrm{M} 2}=0.043-0.2$ than at $\phi_{\mathrm{M} 2}=0.4-0.8$, based on the higher values of normalized matrix_2 concentration at the edge of the grafted layer for ternary PNCs with $\phi_{\mathrm{M} 2}=0.043-0.2$ than at $\phi_{\mathrm{M} 2}=0.4-0.8$; this suggests an optimal concentration of matrix_2 chains for better compatibilization. 

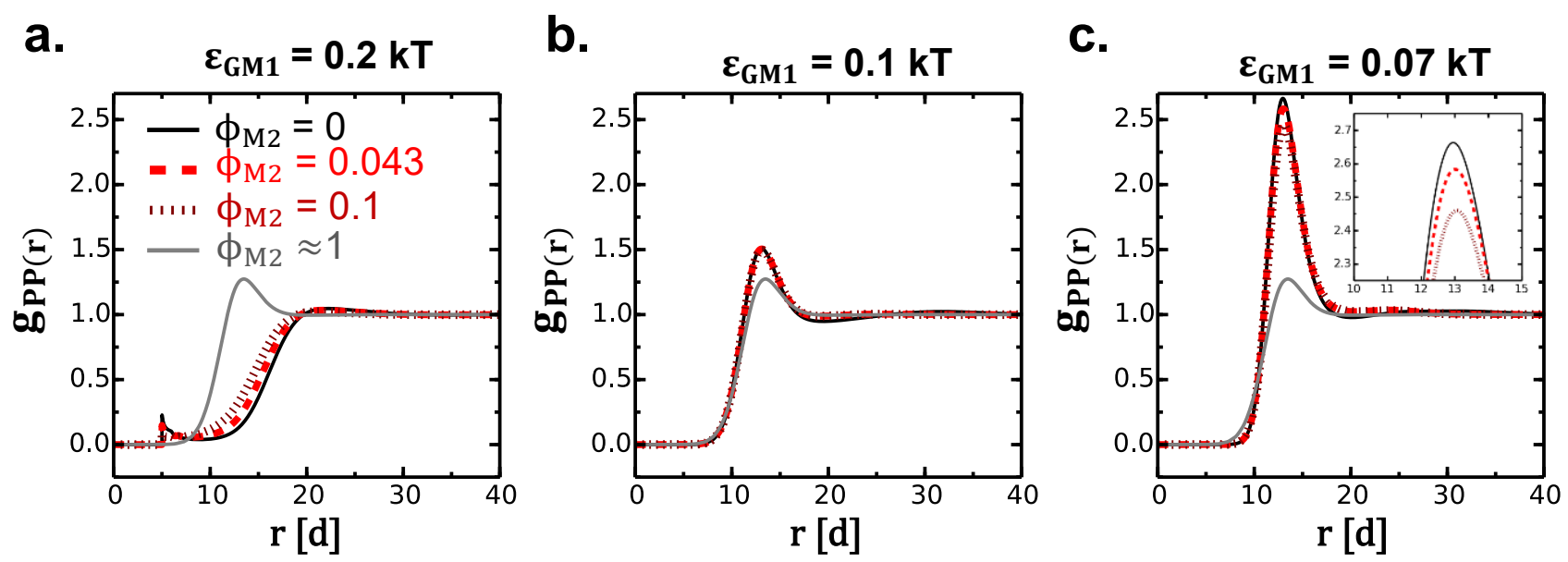

\section{$\phi_{P G P}=0.039$}

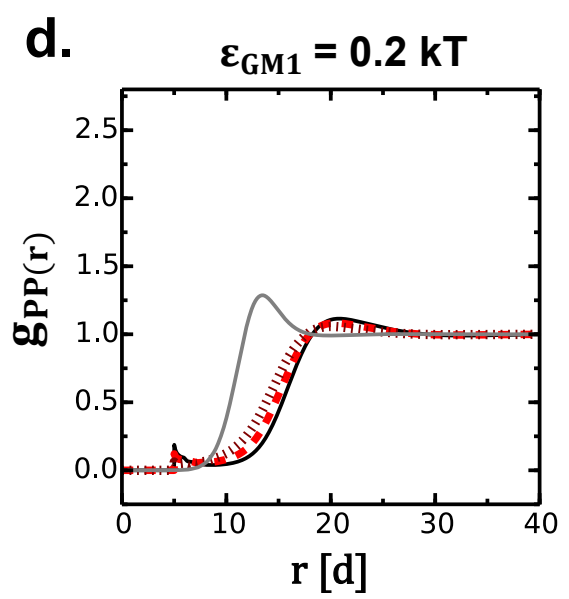

e.
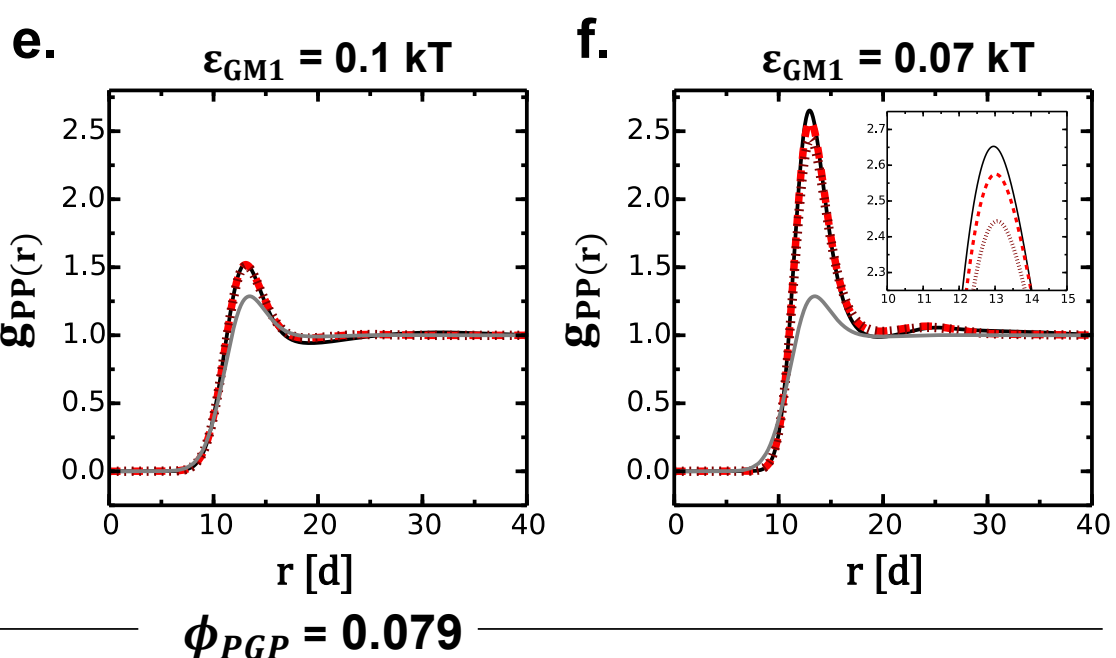

$\phi_{P G P}=0.079$

Figure 9. Particle-particle pair correlation function, $\mathrm{g}_{\mathrm{PP}}(\mathrm{r})$, obtained using PRISM theory for PNCs with $\phi_{\mathrm{PGP}}$ $=0.039(\mathrm{a}-\mathrm{c})$ and, $\phi_{\mathrm{PGP}}=0.079(\mathrm{~d}-\mathrm{f})$ with $\varepsilon_{\mathrm{GM} 1}=0.2 \mathrm{kT}(\mathrm{a}, \mathrm{d}), \varepsilon_{\mathrm{GM} 1}=0.1 \mathrm{kT}(\mathrm{b}, \mathrm{e})$ and $\varepsilon_{\mathrm{GM} 1}=0.07 \mathrm{kT}(\mathrm{c}, \mathrm{f})$. Varying matrix_2 volume fractions are shown using colored lines with $\phi_{\mathrm{M} 2}=0$ (black line), $\phi_{\mathrm{M} 2}=0.043$ (red dashed line), $\phi_{\mathrm{M} 2}=0.1$ (dark red dotted line) and $\phi_{\mathrm{M} 2} \approx 1$ (gray line). The legend in part (a) applies to all parts in this figure and the insets in part c) and part f) have same axes labels as the main plots. These results are for $\varepsilon_{\mathrm{GG}}=\varepsilon_{\mathrm{M} 1 \mathrm{M} 1}=0.1 \mathrm{kT}, \mathrm{D}_{\mathrm{P}}=5 \mathrm{~d}, \mathrm{~N}_{\mathrm{G}}=15, \mathrm{~N}_{\mathrm{M} 1}=75, \mathrm{~N}_{\mathrm{M} 2}=15$, and $\Sigma=0.76$ chains $/ \mathrm{d}^{2}$.

Going beyond the MD simulation results of the graft/matrix interface at low $\phi_{\mathrm{PGP}}$, PRISM theory calculations show the impact of varying the grafted nanoparticle and PMMA 
compatibilizer loadings $\left(\phi_{\mathrm{PGP}}\right.$ and $\left.\phi_{\mathrm{M} 2}\right)$ on the PNC morphology. The chosen $\phi_{\mathrm{PGP}}$ cases of 0.039 and 0.079 correspond to PNCs with 5/95 and 10/90 PMMA-NP/SAN wt.\% in experiments (see Table S2), respectively. Figure 9 shows a series of particle-particle pair correlation functions, $g_{P P}(r)$, as a function of $\phi_{\mathrm{M} 2}$ and $\phi_{\mathrm{PGP}}$.

At $\varepsilon_{\mathrm{GM} 1}=0.2 \mathrm{kT}$ (Figure 9a), as $\phi_{\mathrm{M} 2}$ increases, the $\mathrm{g}_{\mathrm{PP}}(\mathrm{r})$ shifts to lower particle separation distances and exhibits a peak. This is because as $\phi_{\mathrm{M} 2}$ increases, the volume fraction of matrix_1 chains that interact favorably with graft chains decreases, resulting in a slight decrease in the brush layer thickness (seen in brush height values plotted in Figure S12) and smaller interparticle distances. This is also confirmed by corresponding $\mathrm{g}_{\mathrm{PP}}(\mathrm{r})$ plots at intermediate $\phi_{\mathrm{M} 2}$ values between 0 and 1 shown in Figure S15a.

At $\varepsilon_{\mathrm{GM} 1}=0.1 \mathrm{kT}$ (Figure $9 \mathrm{~b}$ ), when the energetic interactions between both graft and matrix_1 and graft and matrix_2 are same, the g $\mathrm{gP}_{\mathrm{PP}}(\mathrm{r})$ peak heights are larger than at $\varepsilon_{\mathrm{GM} 1}=0.2$ $\mathrm{kT}$, indicating reduced particle dispersion. At this $\varepsilon_{\mathrm{GM} 1}, \mathrm{~g}_{\mathrm{PP}}(\mathrm{r})$ profiles are also similar in PNCs with $\phi_{\mathrm{M} 2} \leq 0.1$, where the majority of the matrix is made of the longer matrix_1 chains. There is no difference between the $\mathrm{gPP}_{\mathrm{PP}}(\mathrm{r})$ profiles for the binary $\mathrm{PNC}\left(\phi_{\mathrm{M} 2}=0\right)$ and ternary PNC with low $\phi_{\mathrm{M} 2}$. Figure $\mathrm{S} 15 \mathrm{~b}$ shows that the $\mathrm{g}_{\mathrm{PP}}(\mathrm{r})$ peak height gradually decreases with increasing $\phi_{\mathrm{M} 2}$ values since the volume fraction of the longer matrix_1 chain is reduced relative to shorter matrix_2 chains in the PNC.

At $\varepsilon_{\mathrm{GM} 1}=0.07 \mathrm{kT}$ (Figure 9c), the shapes of the $\mathrm{g}_{\mathrm{PP}}(\mathrm{r})$ exhibit stronger particle aggregation than at higher $\varepsilon_{\mathrm{GM} 1}$ and the $\mathrm{g}_{\mathrm{PP}}(\mathrm{r})$ peak heights for the ternary PNCs with $\phi_{\mathrm{M} 2}=$ 0.043 and 0.1 are slightly smaller (see Figure $9 \mathrm{c}$ inset) than the binary case with $\phi_{\mathrm{M} 2}=0$. This indicates reduced aggregation of the particles in the ternary PNCs with low $\phi_{\mathrm{M} 2}$ than binary PNCs without the compatibilizer, as seen in experiments. 
Upon increasing the grafted filler fraction to $\phi_{\mathrm{PGP}}=0.079$ (Figures 9d-f), we find similar trends as those highlighted so far with $\phi_{\mathrm{PGP}}=0.039$ (Figures 9a-c). Particularly, at higher temperatures with increasing loadings of matrix_2 chains (Figures 9f and S15f), the grafted nanoparticles are more dispersed than the binary PNC with $\phi_{\mathrm{M} 2}=0$.

Taken together, the results in Figure 9 suggest that at low temperatures (below the experimental LCST), the miscibility in ternary PNCs comprised of chemically dissimilar graft (PMMA) and majority matrix (SAN) polymers with a small amount of compatibilizer (chemically identical matrix (PMMA)) is greater than in binary PNCs with no compatibilizer. This confirms the role of the PMMA chains in the ternary PNCs in stabilizing the interface between graft and matrix chains. We note that at elevated temperatures above the LCST for PMMA/SAN (or due to the generality of the model, at temperatures below the UCST for a UCST graft-matrix system), the PNC miscibility in ternary PNCs comprised of chemically dissimilar graft and majority matrix polymers with a small amount of chemically identical matrix is lower than in a binary PNC with chemically identical and chain-length matched graft and matrix polymers. 

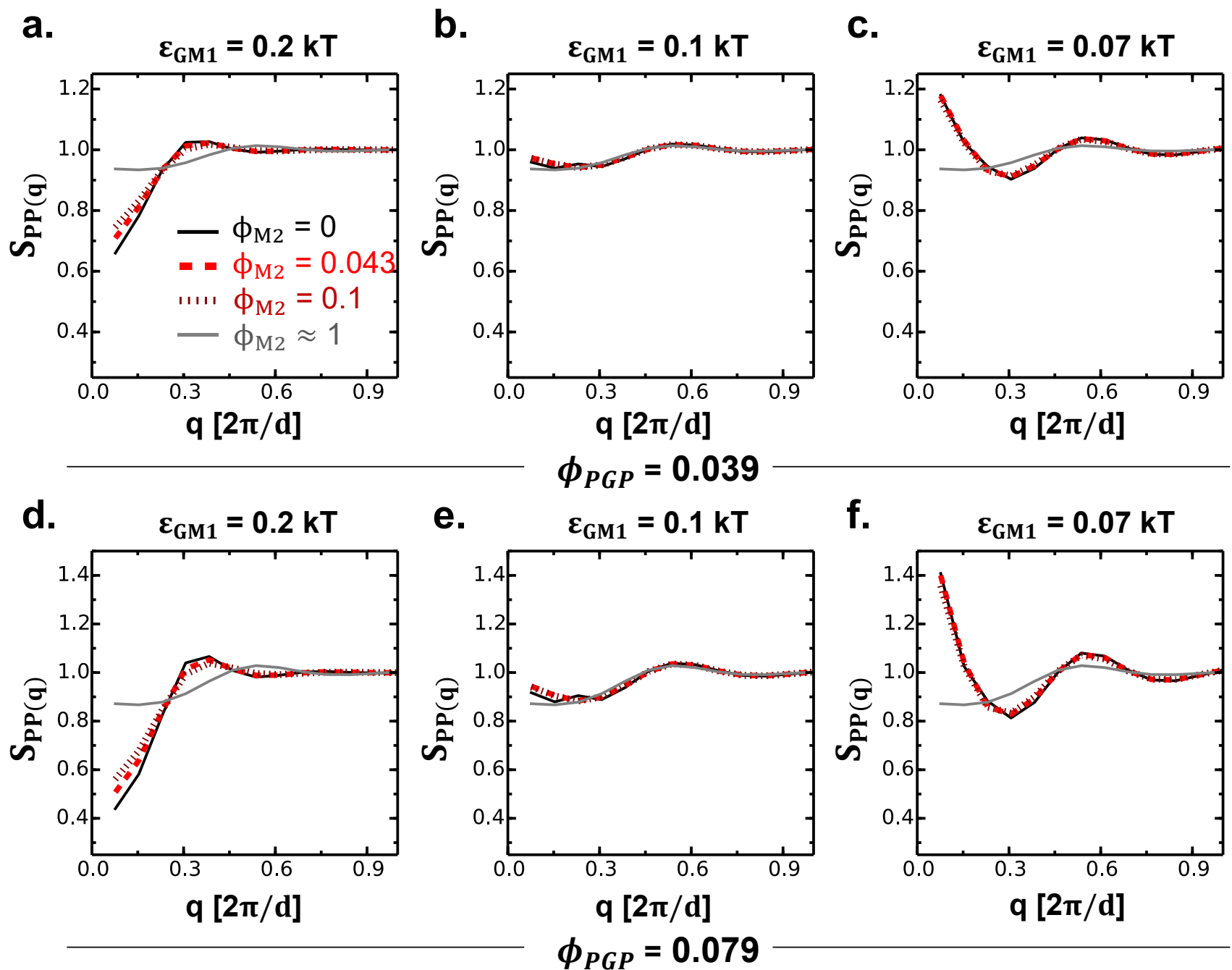

Figure 10. Particle-particle structure factor, $\mathrm{S}_{\mathrm{PP}}(\mathrm{q})$ for $\mathrm{PNCs}$ with $\phi_{\mathrm{PGP}}=0.039$ (a-c) and, $\phi_{\mathrm{PGP}}=0.079$ (d-f) with $\varepsilon_{\mathrm{GM} 1}$ $=0.2 \mathrm{kT}(\mathrm{a}$ and $\mathrm{d}), \varepsilon_{\mathrm{GM} 1}=0.1 \mathrm{kT}(\mathrm{b}$ and $\mathrm{e})$ and $\varepsilon_{\mathrm{GM} 1}=0.07 \mathrm{kT}(\mathrm{c}$ and $\mathrm{f})$. Varying matrix 2 volume fractions are shown using colored lines with $\phi_{\mathrm{M} 2}=0$ (black line), $\phi_{\mathrm{M} 2}=0.043$ (red dashed line), $\phi_{\mathrm{M} 2}=0.1$ (dark red dotted line) and $\phi_{\mathrm{M} 2} \approx$ 1 (gray line). The legend in part (a) applies to all parts in this figure. These results are for $\varepsilon_{\mathrm{GG}}=\varepsilon_{\mathrm{M} 1 \mathrm{M} 1}=0.1 \mathrm{kT}, \mathrm{D}_{\mathrm{P}}=$ $5 \mathrm{~d}, \mathrm{~N}_{\mathrm{G}}=15, \mathrm{~N}_{\mathrm{M} 1}=75, \mathrm{~N}_{\mathrm{M} 2}=15$, and $\Sigma=0.76$ chains $/ \mathrm{d}^{2}$.

Lastly, we confirm that despite the differences between binary and ternary PNCs in the particle-particle correlations elucidated by the $\mathrm{g}_{\mathrm{PP}}(\mathrm{r})$ in Figure 9, as seen in experiments at high temperatures (Figures 2 and 3), we do not see major differences in the macroscale morphologies within the binary and ternary PNC with low $\phi_{\mathrm{M} 2}$ (Figure 10). The only differences we see 
between the binary and ternary PNCs at low $\phi_{\mathrm{M} 2}$ are at high $\varepsilon_{\mathrm{GM} 1}$ of $0.2 \mathrm{kT}$; for example, in Figure 10a, $S_{P P}(q \rightarrow 0)$ increases with increasing $\phi_{\mathrm{M} 2}$ (also seen in Figure S16a). The binary PNC containing only PMMA chains $\left(\phi_{\mathrm{M} 2} \approx 1\right)$ exhibits a slight upturn at low q and has the largest $\mathrm{S}_{\mathrm{PP}}(\mathrm{q} \rightarrow 0)$ magnitude. This suggests that at high values of $\varepsilon_{\mathrm{GM} 1}$, the favorable enthalpic interactions between graft and matrix_1 chains in both binary and ternary PNCs serve to better disperse the NPs than the purely entropic forces between graft and matrix_ 2 chains. At $\varepsilon_{\mathrm{GM} 1}=$ 0.07 kT (Figure 10c), binary and ternary PNCs with $\phi_{\mathrm{M} 2}=0-0.1$ all show a significant upturn as $\mathrm{q} \rightarrow 0$, which reflects NP aggregation.

Overall, results from PRISM theory and MD simulations support the experimental observations that small amounts of matrix_2 (PMMA) chains serve as interfacial compatibilizers between the PMMA graft and long matrix_1 (SAN) chains at high temperature. This interfacial compatibilization reduces the particle-particle correlation within aggregates likely explaining the reduction in cluster sizes of the nanoparticles at high temperatures.

\section{CONCLUSION}

In this study, binary and ternary phase diagrams were experimentally mapped using a combination of cloud point observations, TEM, and SAXS for PMMA-NP/SAN and PMMANP/SAN/PMMA composites, respectively. When compared, they illustrate that the addition of a ternary component, namely, PMMA homopolymer to the binary PMMA-NP/SAN composite, results in an increased miscibility window at low loadings of grafted nanoparticles. The improved miscibility from the added homopolymer can be understood as a result of an interfacial compatibilization between the SAN matrix and grafted PMMA brush due to the PMMA homopolymer localization. A simple geometric model is presented that predicts the extent of homopolymer compatibilization based on the relative loadings of each constituent. In addition, 
using PRISM theory calculations and MD simulations, the compatibilization effect of the added PMMA chains is systematically probed and compared to the experimental results. Importantly, MD simulations confirm the role of the PMMA chains in stabilizing the interface between graft and matrix chains in ternary PNCs. Additionally, PRISM theory results predict that the extent of compatibilization can be further tuned by varying the composition of the ternary PNCs. The generality of this compatibilization effect should apply to any PNC where the compatibilizer wets the grafted nanoparticle such that it reduces the interfacial energy between the binary constituents. Further, we hope that these results motivate further experimental and theoretical studies aimed at exploring the entire parameter space (i.e. molecular weight, grafting density, composition, etc.) in ternary nanocomposites to determine the parameters that yield the greatest improvement in miscibility. This study serves as a guideline to facilitate PNC miscibility at elevated temperatures, which is a critical parameter in designing technological applications, such as organic devices, that operate at high temperatures. 


\section{ACKNOWLEDGEMENTS}

S.M.M., N.M.K., C.R.B., and R.J.C. were supported by the National Science Foundation Partnerships for International Research and Education Program (NSF-PIRE), Grant \#1545884. This research was additionally supported by the POLYMERS-DMR1905912 (S.M.M., N.M.K, C.R.B, R.J.C.) and MRSEC-DMR-1720530 (S.M.M., N.M.K., C.R.B, R.J.C.) programs. S.M.M., M.M., and P.R. also acknowledge support from the Centre National de la Recherche Scientifique (CNRS) at the laboratory of "Systèmes Moléculaires et nanoMatériaux pour l'Énergie et la Santé", (UMR5819-SyMMES (CNRS/CEA/Univ. Grenoble Alpes), Grenoble, France) and funding from the Agence Nationale de la Recherche (ANR): ANR-15- PIRE-000101 and ANR-15-PIRE-0001-07. The authors acknowledge use of the Duel Source and Environmental X-ray Scattering facility operated by the Laboratory for Research on the structure of Matter at the University of Pennsylvania (NSF MRSEC 17-20530). The equipment purchase was made possible by a NSF MRI grant (17-25969), an ARO DURIP grant (W911NF-17-10282), and the University of Pennsylvania. We thank Dr. Karen I. Winey and Dr. Christopher B. Murray for use of their laboratory's ultramicrotome and TEM, respectively. A.K. and A.J. acknowledge funding from U.S. Department of Energy, Office of Science Grant (DESC0017753) and the use of Informational Technologies (IT) resources at the University of Delaware, including the high performance computing resources of the Farber and Caviness supercomputing clusters. The authors also acknowledge Dr. Robert A. Riggleman and Dr. Daeyeon Lee for helpful discussions. 


\section{SUPPORTING INFORMATION}

Characterization of PNC constituents, sample preparation for ultramicrotomy, as-cast PNC characterization, cloud point images, TEM images of the PNCs annealed at longer times, discussion of SAXS model fitting, SAXS profiles at different NP loadings, simulation/theory parameters, brush height profiles, concentration profiles for SAN matrix at different interaction strengths, additional concentration profiles for PMMA graft and PMMA matrix chains at different loadings of PMMA matrix, additional $\mathrm{g}_{\mathrm{PP}}(\mathrm{r})$ and $\mathrm{S}_{\mathrm{PP}}(\mathrm{q})$ at different loadings of PMMA matrix, and $\mathrm{S}_{\mathrm{GG}}(\mathrm{q})$ for PNCs at different compositions.

\section{AUTHOR INFORMATION}

*S.M.M and N.M.K. contributed equally to this work.

\section{REFERENCES}

(1) Glynos, E.; Papoutsakis, L.; Pan, W.; Giannelis, E. P.; Nega, A. D.; Mygiakis, E.; Sakellariou, G.; Anastasiadis, S. H. Nanostructured Polymer Particles as Additives for High Conductivity, High Modulus Solid Polymer Electrolytes. Macromolecules 2017, 50 (12), 4699-4706. https://doi.org/10.1021/acs.macromol.7b00789.

(2) Glynos, E.; Petropoulou, P.; Mygiakis, E.; Nega, A. D.; Pan, W.; Papoutsakis, L.; Giannelis, E. P.; Sakellariou, G.; Anastasiadis, S. H. Leveraging Molecular Architecture to Design New, All-Polymer Solid Electrolytes with Simultaneous Enhancement in Modulus and Ionic Conductivity. Macromolecules 2018, 51 (7), 2542-2550. https://doi.org/10.1021/acs.macromol.7b02394.

(3) Maréchal, M.; Niepceron, F.; Gebel, G.; Mendil-Jakani, H.; Galiano, H. Inside the Structure of a Nanocomposite Electrolyte Membrane: How Hybrid Particles Get along with the Polymer Matrix. Nanoscale 2015, 7 (7), 3077-3087. https://doi.org/10.1039/c4nr05330c.

(4) Hore, M. J. A.; Composto, R. J. Nanorod Self-Assembly for Tuning Optical Absorption. 
ACS Nano 2010, 4 (11), 6941-6949. https://doi.org/10.1021/nn101725j

(5) Hore, M. J. A.; Composto, R. J. Functional Polymer Nanocomposites Enhanced by Nanorods. Macromolecules 2014, 47 (3), 875-887. https://doi.org/10.1021/ma402179w.

(6) Ferrier, R. C.; Lee, H. S.; Hore, M. J. A.; Caporizzo, M.; Eckmann, D. M.; Composto, R. J. Gold Nanorod Linking to Control Plasmonic Properties in Solution and Polymer Nanocomposites. Langmuir 2014, 30 (7), 1906-1914. https://doi.org/10.1021/la404588w.

(7) Bilchak, C. R.; Buenning, E.; Asai, M.; Zhang, K.; Durning, C. J.; Kumar, S. K.; Huang, Y.; Benicewicz, B. C.; Gidley, D. W.; Cheng, S.; Sokolov, A. P.; Minelli, M.; Doghieri, F. Polymer-Grafted Nanoparticle Membranes with Controllable Free Volume. $\begin{array}{llll}\text { Macromolecules } & \mathbf{2 0 1 7}, & 50 & \text { (18), }\end{array}$ https://doi.org/10.1021/acs.macromol.7b01428.

(8) Ginzburg, V. V. Influence of Nanoparticles on Miscibility of Polymer Blends. A Simple Theory. Macromolecules 2005, 38 (6), 2362-2367. https://doi.org/10.1021/ma0482821.

(9) Ferrier, R. C.; Koski, J.; Riggleman, R. A.; Composto, R. J. Engineering the Assembly of Gold Nanorods in Polymer Matrices. Macromolecules 2016, 49 (3), 1002-1015. https://doi.org/10.1021/acs.macromol.5b02317.

(10) Krook, N. M.; Ford, J.; Marechal, M.; Rannou, P.; Meth, J. S.; Murray, C. B.; Composto, R. J. Alignment of Nanoplates in Lamellar-Forming Diblock Copolymer Domains. ACS Macro Lett. 2018, 7 (12), 1400-1407. https://doi.org/10.1021/acsmacrolett.8b00665

(11) Rasin, B.; Chao, H.; Jiang, G.; Wang, D. Soft Matter Dispersion and Alignment of Nanorods in Cylindrical Block Copolymer Thin Films. Soft Matter 2016, 12 (7), 21772185. https://doi.org/10.1039/c5sm02442k.

(12) Glynos, E.; Petropoulou, P.; Mygiakis, E.; Nega, A. D.; Pan, W.; Papoutsakis, L.; Giannelis, E. P.; Sakellariou, G.; Anastasiadis, S. H. Leveraging Molecular Architecture To Design New, All-Polymer Solid Electrolytes with Simultaneous Enhancement in Modulus and Ionic Conductivity. Macromolecules 201851 (7), 2542-2550. https://doi.org/10.1021/acs.macromol.7b02394.

(13) Schmitt, M.; Zhang, J.; Lee, J.; Lee, B.; Ning, X.; Zhang, R.; Karim, A.; Davis, R. F.; Matyjaszewski, K.; Bockstaller, M. R. Polymer Ligand-Induced Autonomous Sorting and Reversible Phase Separation in Binary Particle Blends. Sci. Adv. 2016, 2 (12), e1601484. https://doi.org/10.1126/sciadv.1601484. 
(14) Zhang, R.; Lee, B.; Bockstaller, M. R.; Kumar, S. K.; Stafford, C. M.; Douglas, J. F.; Raghavan, D.; Karim, A. Pattern-Directed Phase Separation of Polymer-Grafted Nanoparticles in a Homopolymer Matrix. Macromolecules 2016, 49 (10), 3965-3974. https://doi.org/10.1021/acs.macromol.6b00228.

(15) Higashida, N.; Kressler, J.; Inoue, T. . Lower critical solution temperature and upper critical solution temperature phase behaviour in random copolymer blends: poly(styreneco-acrylonitrile)/poly(methyl methacrylate) and poly(styrene-co-acrylonitrile)/poly( $\varepsilon$ caprolactone). Polymer 1995, 36 (14), 2161-2164. https://doi.org/10.1016/00323861(95) $93654-5$

(16) Suess, M.; Kressler, J.; Kammer, H. W. The Miscibility Window of Poly(Methyl Methacrylate)/Poly(Styrene-Co-Acrylnitrile) Blends. Polymer 1987, 28 (6), 957-960. https://doi.org/10.1016/0032-3861(87)90169-8

(17) Cowie, J. M. G.; Reid, V. M. C.; McEwen, I. J. Prediction of the Miscibility Range in Blends of Poly(Styrene-Co-Acrylonitrile) and Poly(N-Phenyl Itaconimide-Co-Methyl Methacrylate): A Six-Interaction-Parameter System. Polymer 1990, 31 (3), 486-489. https://doi.org/10.1016/0032-3861(90)90390-K.

(18) Nishimoto, M.; Keskkula, H.; Pault, D. R. Miscibility of Blends of Polymers Based on Styrene, Acrylonitrile and Methyl Methacrylate. Polymer 1989, 30 (7), 1279-1286. https://doi.org/10.1016/0032-3861(89)90049-9

(19) Fowler, M. E.; Barlow, J. W.; Paul, D. R. Effect of Copolymer Composition on the Miscibility of Blends of Styrene-acrylonitrile Copolymers with Poly(Methyl Methacrylate). Polymer 1986, 28 (7), 1177-1184. https://doi.org/10.1016/00323861(87)90261-8

(20) Liu, J.; Gao, Y.; Cao, D.; Zhang, L.; Guo, Z. Nanoparticle Dispersion and Aggregation in Polymer Nanocomposites: Insights from Molecular Dynamics Simulation. Langmuir 2011, 27 (12), 7926-7933. https://doi.org/10.1021/la201073m

(21) Gao, J.; Huang, C.; Wang, N.; Yu, W.; Zhou, C. Phase Separation of Poly(Methyl Methacrylate)/Poly(Styrene-Co-Acrylonitrile) Blends in the Presence of Silica $\begin{array}{lllll}\text { Nanoparticles. } & \text { Polymer } & \text { 2012, } & 53 & \text { (8), }\end{array}$ https://doi.org/10.1016/j.polymer.2012.02.027.

(22) Gam, S.; Corlu, A.; Chung, H.-J.; Ohno, K.; Hore, M. J. A.; Composto, R. J. A Jamming 
Morphology Map of Polymer Blend Nanocomposite Films. Soft Matter 2011, 7 (16), 7262-7268. https://doi.org/10.1039/c1sm05619k.

(23) Chung, H. J.; Kim, J.; Ohno, K.; Composto, R. J. Controlling the Location of Nanoparticles in Polymer Blends by Tuning the Length and End Group of Polymer Brushes. ACS Macro Lett. 2012, 1 (1), 252-256. https://doi.org/10.1021/mz200068p.

(24) Chung, H. J.; Ohno, K.; Fukuda, T.; Composto, R. J. Self-Regulated Structures in Nanocomposites by Directed Nanoparticle Assembly. Nano Lett. 2005, 5 (10), 1878-1882. https://doi.org/10.1021/n1051079e.

(25) Li, W. Effect of Silica Nanoparticles on the Morphology of Polymer Blends. Technische Universiteit Eindhoven. 2011. https://doi.org/10.6100/IR719366.

(26) Ojha, S.; Dang, A.; Hui, C. M.; Mahoney, C.; Matyjaszewski, K.; Bockstaller, M. R. Strategies for the Synthesis of Thermoplastic Polymer Nanocomposite Materials with High Inorganic Filling Fraction. Langmuir 2013, 29 (28), 8989-8996. https://doi.org/10.1021/la401522v.

(27) Martin, T. B.; Mongcopa, K. I. S.; Ashkar, R.; Butler, P.; Krishnamoorti, R.; Jayaraman, A. Wetting-Dewetting and Dispersion-Aggregation Transitions Are Distinct for Polymer Grafted Nanoparticles in Chemically Dissimilar Polymer Matrix. J. Am. Chem. Soc. 2015, 137 (33), 10624-10631. https://doi.org/10.1021/jacs.5b05291.

(28) Ohno, K.; Morinaga, T.; Koh, K.; Tsujii, Y.; Fukuda, T. Synthesis of Monodisperse Silica Particles Coated with Well-Defined, High-Density Polymer Brushes by Surface-Initiated Atom Transfer Radical Polymerization. Macromolecules 2005, 38 (6), 2137-2142. https://doi.org/10.1021/ma048011q.

(29) Grest, G. S.; Kremer, K. Molecular Dynamics Simulation for Polymers in the Presence of a Heat Bath. Phys. Rev. A 1986, 33 (5), 3628(R). https://doi.org/10.1103/PhysRevA.33.3628

(30) Jones, J. E. On the Determination of Molecular Fields.-II. From the Equation of State of a Gas. Proc. R. Soc. London, Ser. A 1924, 106 (738), 463-477. https://doi.org/10.1098/rspa.1924.0082

(31) Weeks, J. D.; Chandler, D.; Andersen, H. C. Role of Repulsive Forces in Determining the Equilibrium Structure of Simple Liquids. J. Chem. Phys. 1971, 54 (12), 5237-5247. https://doi.org/10.1063/1.1674820 
(32) Plimpton, S. Fast Parallel Algorithms for Short-Range Molecular Dynamics. J. Comput. Phys. 1995, 117 (1), 1-19. https://doi.org/10.1006/jcph.1995.1039

(33) Nosé, S. A Molecular Dynamics Method for Simulations in the Canonical Ensemble. Mol. Phys. 1984, 52 (2), 255-268. https://doi.org/10.1080/00268978400101201

(34) Hoover, W. G. Canonical Dynamics: Equilibrium Phase-Space Distributions. Phys. Rev. A 1985, 31 (3), 1695. https://doi.org/10.1103/PhysRevA.31.1695

(35) Schweizer, K. S.; Curro, J. G. PRISM Theory of the Structure, Thermodynamics, and Phase Transitions of Polymer Liquids and Alloys. In Atomistic Modeling of Physical Properties; Monnerie, L., Suter, U. W., Eds.; Springer: Berlin, 1994; Vol. 116, pp 319377.

(36) Schweizer, K. S.; Curro, J. G. Integral-Equation Theory of the Structure of Polymer Melts. Phys. Rev. Lett. 1987, 58 (3), 246. https://doi.org/10.1103/PhysRevLett.58.246

(37) Schweizer, K. S.; Curro, J. G. Integral Equation Theories of the Structure, Thermodynamics, and Phase Transitions of Polymer Fluids. Adv. Chem. Phys. 1997, 98, 1-142. https://doi.org/10.1002/9780470141571.ch1

(38) Ornstein, L. S.; Zernike, F. Accidental Deviations of Density and Opalescence at the Critical Point of a Single Substance. Proc. Akad. Sci. 1914, 17 (2), 793-806.

(39) Hansen, J.-P.; McDonald, I. R. Theory of Simple Liquids, 2nd ed.; Academic Press: London, 1986.

(40) Hooper, J. B.; Schweizer, K. S. Theory of Phase Separation in Polymer Nanocomposites. Macromolecules 2006, 39 (15), 5133-5142. https://doi.org/10.1021/ma060577m

(41) Hooper, J. B.; Schweizer, K. S.; Desai, T. G.; Koshy, R.; Keblinski, P. Structure, Surface Excess and Effective Interactions in Polymer Nanocomposite Melts and Concentrated Solutions. J. Chem. Phys. 2004, 121 (14), 6986-6997. https://doi.org/10.1063/1.1790831

(42) Chandler, D.; Andersen, H. C. Optimized Cluster Expansions for Classical Fluids. II. Theory of Molecular Liquids. J. Chem. Phys. 1972, 57 (5), 1930-1937. https://doi.org/10.1063/1.1678513

(43) Martin, T. B.; Gartner III, T. E.; Jones, R. L.; Snyder, C. R.; Jayaraman, A. PyPRISM: A Computational Tool for Liquid-State Theory Calculations of Macromolecular Materials. Macromolecules 2018, 51 (8), 2906-2922. https://doi.org/10.1021/acs.macromol.8b00011

(44) Gam, S.; Meth, J. S.; Zane, S. G.; Chi, C.; Wood, B. A.; Seitz, M. E.; Winey, K. I.; 
Clarke, N.; Composto, R. J. Macromolecular Diffusion in a Crowded Polymer Nanocomposite. $\quad$ Macromolecules 2011, $44 \quad$ (9), 3494-3501. https://doi.org/10.1021/ma102463q.

(45) Meth, S.; Zane, S. G.; Chi, C.; Londono, J. D.; Wood, B. A.; Cotts, P.; Keating, M.; Guise, W.; Weigand, S. Development of Filler Structure in Colloidal Silica-Polymer Nanocomposites. $\quad$ Macromolecules 2011, $44 \quad$ (20), 8301-8313. https://doi.org/10.1021/ma201714u

(46) Newby, B. Z.; Composto, R. J. Influence of Lateral Confinement on Phase Separation in Thin Film Polymer Blends. Macromolecules 2000, 33 (9), 3274-3282. https://doi.org/10.1021/ma992092m

(47) Akcora, P.; Kumar, S. K.; Li, Y.; Benicewicz, B. C.; Schadler, L. S. Segmental Dynamics in PMMA-Grafted Nanoparticle Composites. Macromolecules 2010, 43 (19), 8275-8281. https://doi.org/10.1021/ma101240j.

(48) Akcora, P.; Liu, H.; Kumar, S. K.; Moll, J.; Li, Y.; Benicewicz, B. C.; Schadler, L. S.; Acehan, D.; Panagiotopoulos, A. Z.; Pryamitsyn, V.; Ganesan, V.; Ilavsky, J.; Thiyagarajan, P.; Colby, R. H.; Douglas, J. F. Anisotropic Self-Assembly of Spherical Polymer-Grafted Nanoparticles. Nat. Mater. 2009, $8 \quad$ (4), 354-359. https://doi.org/10.1038/NMAT2404.

(49) Chung, H.; Wang, H.; Composto, R. J. A Morphology Map Based on Phase Evolution in Polymer Blend Films. Macromolecules 2006, $39 \quad$ (1), 153-161. https://doi.org/10.1021/ma051513z

(50) Roe, R. J.; Lu, L. R. Effect of Polydispersity on the Cloud-Point Curves of Polymer Mixtures. J. Polym. Sci. B Polym. Phys. 1985, 23 (5), 917-924. https://doi.org/10.1002/pol.1985.180230506.

(51) Rubinstein, M; Colby, R. H. Polymer Physics, Oxford University Press, Oxford, UK, 2003.

(52) Martin, T. B.; Jayaraman, A. Effect of Matrix Bidispersity on the Morphology of Polymer-grafted Nanoparticle-filled Polymer Nanocomposites. J. Polym. Sci. Part B Polym. Phys. 2014, 52 (24), 1661-1668. https://doi.org/10.1002/polb.23517 